\title{
Control of Social Withdrawal of Mice Deficient for the Autism Gene Magel2 by Restoration of Vasopressin-Oxytocin Dialogue in Septum
}

\author{
BORIE Amélie M. ${ }^{1}$, DROMARD Yann ${ }^{1}$, DUFNER Djodi ${ }^{1}$, POLLOZI Emi ${ }^{1}$, HUZARD \\ Damien $^{1}$, TÖMBÖLI Csaba ${ }^{2}$, OLMA Aleksandra ${ }^{3}$, MANNING Maurice ${ }^{3}$, COLSON Pascal ${ }^{1,4}$, \\ GUILLON Gilles ${ }^{1}$, MUSCATELLI Françoise ${ }^{5}$, DESARMÉNIEN Michel G. ${ }^{1, *} \&$ \\ JEANNETEAU Freddy ${ }^{1, *,}$
}

1 Institut de Génomique Fonctionnelle, INSERM, CNRS, Montpellier University, France

2 Laboratory of Chemical Biology, Biological Research Centre, Szeged, Hungary

3 University of Toledo College of Medicine and Life Sciences, USA

$4 \quad$ Department of Anesthesiology and Critical Care Medicine, Arnaud de Villeneuve Academic Hospital, Montpellier, France.

5 Institut des Neurosciences de la Méditerranée, INSERM, Aix-Marseille University, France

$* \quad$ Equal contribution
freddy.jeanneteau@,igf.cnrs.fr 


\section{Summary}

Intellectual and social disabilities are common comorbidities in adolescents and adults with Magel2 gene deficiency characterizing the Prader-Willi and Schaaf-Yang neurodevelopmental syndromes. The cellular and molecular mechanisms underlying the risk for autism in these syndromes are unexplored. Here we used Magel2 knockout mice combined with optogenetic/pharmacological tools to characterize disease modifications in the social brain network. We find that the degree of social novelty moderates a dialogue between vasopressin and oxytocin in the lateral septum, a region organizing sequential content of sensory experiences. Social withdrawal of mice lacking Magel2 is alleviated by restoration of dialoguelead by vasopressin. This preclinical study identifies the collective actions of vasopressin and oxytocin in the lateral septum as a key factor in the pathophysiology.

\section{Introduction}

Autism Spectrum Disorders (ASD) affect 1 in 68 and are characterized by difficulties with communication, restrictive interests and repetitive behaviors influencing the ability to function properly (Diagnostic and Statistical Manual of Mental Disorders, 5th edition). Treatment of ASD is marginal such that management of disability-adjusted life years imposes substantial economic cost and burden on families and society ${ }^{1}$. Neurodevelopmental disorders characterized by mutations of chromosome 15q11-13 exhibit higher than normal risk for comorbid ASD, indicating the importance of genes in this locus for the pathophysiology ${ }^{2,3}$. It is a large chromosomal deletion of 15q11-13 in Prader-Willi Syndrome (PWS) and a disruption of one gene in this locus, Magel2, in Schaaf-Yang Syndrome (SYS) that provide respectively, 25\% and $75 \%$ risk for ASD based on clinical assessment by an expert physician ${ }^{2,3}$. Magel2 is a maternally imprinted, paternally expressed gene central to the pathophysiology of PWS and $\mathrm{SYS}^{4}$, which deficiency interferes with developmental functions essential for setting multi-scale organization of the nervous system controlling muscle tone and feeding ${ }^{5,6}$. Common symptomatic features of PWS and SYS are hypotonia, feeding difficulties during early life, social withdrawal, intellectual and/or developmental delay ${ }^{4,7,8}$.

Mice lacking Magel2 gene are a good model of SYS and PWS with construct and face validity as knockout mice present hypotonia, feeding difficulties during early life and social deficits $^{9-11}$. Treatment with oxytocin (OXT) around birth restores feeding in Magel2 knockout (KO) mice ${ }^{9}$ and pediatric PWS patients ${ }^{12}$ suggesting predictive validity of this mouse model. Adult Magel2 KO mice also present ASD-like features, such as social withdrawal ameliorated by perinatal OXT treatments ${ }^{9,10}$.

Modulation of social behavior with OXT has been at the center of many studies ${ }^{13}$ and it is now accepted that OXT contributes to filtering social salience signals ${ }^{14}$. OXT is used in numerous clinical trials ${ }^{15}$ with promising results for the treatment of ASD. While beneficial effects were observed in PWS patients treated with OXT, these effects are age dependent ${ }^{16}$ and not consistently found over different clinical trials ${ }^{17}$. Furthermore, chronic OXT treatments might be deleterious to specific aspects of social behavior ${ }^{18,19}$. This suggests that OXT therapy is not sufficient to treat social disabilities beyond the early postnatal critical period of neurodevelopment ${ }^{20}$ and stresses the need to better understand the OXT system and its modulators for treating social withdrawal in the adults.

Vasopressin (AVP), a peptide sharing many features with $\mathrm{OXT}^{21}$, is also important for the regulation of social salience and has been in the center of new clinical studies. ASD patients improved social communication upon treatment with $\mathrm{AVP}^{22}$ as well as with antagonists of AVP receptor subtype 1a (AVPR1a) ${ }^{23}$. The positive outcomes of both trials demands clarity about the 
mechanisms underlying AVPR responses in ASD and related diseases like PWS and SYS. We hypothesized that collective actions of OXT and AVP in the social salience brain network could explain the controversial efficacy of AVPR targeted therapies. Unfortunately, the roles of OXT and AVP have mainly been interrogated in isolation whereas combinatorial effects are anticipated $^{24}$ given that both OXT and AVP are secreted in the brain upon social encounters ${ }^{25}$. Here, we devised strategies to understand the dual functions of AVP and OXT during social encounters in physiological conditions and in the context of Magel2 deficiency.

\section{Results}

\section{Social withdrawal linked to brain theta rhythmicity defects in $\mathrm{Magel}^{+m /-p}$ mice}

Brain rhythmic activity, notably theta paced, across multiple regions of the social brain network is modulated by the novelty of social stimulus and associated with cognitive and emotional behaviors in humans and rodents ${ }^{26,27}$. Nonetheless, socially evoked theta rhythmicity has not been studied in animal models featuring autistic-like social withdrawal like the Magel2 $2^{+m / p}$ mice. We used a telemetric system to record the brain electroencephalogram (EEG) from wire electrodes chronically implanted atop cortex as previously described ${ }^{28}$. Wired animals were subjected to multiple social trials with an unfamiliar juvenile (T1 to T4) followed by the encounter with a different juvenile (T5) to discriminate between degrees of novelty of the social stimulus (Fig. 1a). Magel $2^{+m /-p}$ mice performed poorly on the discrimination task with a mouse whereas object exploration was normal compared to WT littermate controls (Fig. 1b). At T1, EEG power spectral density analysis showed a socially induced modulation of activity in the theta band of healthy controls that is absent in Magel2 $2^{+m /-p}$ mice (Fig. 1c). The effect of Magel $2^{+m /-p}$ was specific of social stimulus as no difference with controls was observed during trials with objects (Fig. 1c). Changes of socially evoked theta rhythmicity correlated with the exploration time of conspecifics in WT controls but not in Magel2 ${ }^{+m /-p}$ mice (Fig. 1d). This contrasted with theta rhythmicity in trials with objects that did not correlate with the degree of novelty in WT controls but did in Magel2 ${ }^{+m /-p}$ mice (Fig. 1d). Therefore, this behavioral paradigm is sufficiently robust in Magel2 $2^{+m /-p}$ mice to detect social exploration disabilities consistent with brain theta rhythmicity defects particularly marked during the first social encounter.

\section{Abnormal septal oxytocinergic and vasopressinergic systems in Magel2 $^{+m /-p}$ mice}

Previously, we showed that low number of OXTR binding sites specifically in the lateral septum (LS) of Magel2 ${ }^{+m /-p}$ mice covariate with social withdrawal ${ }^{10}$. This suggests a key role for the LS and its modulation by OXT in the physiopathology. C-Fos mapping showed more robust induction in the LS after interaction with a conspecific than with an object (Fig. S1a), an effect validated with p-S6 as indicator of rapid signaling (Fig. S1b). Compared to WT controls, basal expression of c-Fos was high and less reactive to social trials particularly in the LS dorsal (LSD) of Magel2 ${ }^{+m /-p}$ mice (Fig. S1c,d). Additionally, there were fewer and shorter AVP fibers in the LSD of Magel2 ${ }^{+m /-p}$ mice (Fig. S2a,b) as well as more OXT fibers in the septum (Fig. S2c,d). Such genotypic differences of topological innervations in septum suggest that both OXT and AVP actions may influence sociability between mice as previously hypothesized in healthy rats $^{29,30}$.

To monitor the impact of AVP and the OXT analog agonist TGOT on theta rhythmicity, we combined bilateral intraseptal peptide injections with EEG recordings in freely moving cannulated mice (Fig. 2a). EEG power spectral analysis showed a trough of activity in the 4-8 $\mathrm{Hz}$ band and peak of activity in the $8-12 \mathrm{~Hz}$ band with AVP that contrasted with an opposite 
effect of TGOT (Fig. 2b). Such changes of theta rhythmicity mimicked patterns evoked in WT mice at social trials $\mathrm{T} 1$ and T4, respectively ( $c f$. Fig. 1c). Implicitly, it suggests that a deficit of septal AVP at T1 might impair social exploration similar to the effect of Magel2 deficiency. To test this possibility, we injected an AVPR antagonist, the Manning compound (MC) into the septum at $\mathrm{T} 1$ or $\mathrm{T} 3$ to assess its influence on social behavior and theta rhythm (see methods for details about in vivo pharmacology). Only at T1, the blockade of AVPR with MC impaired social exploration like the effect of Magel2 deficiency (Fig. 2c). Similarly, and only at T3, the blockade of OXT receptors (OXTR) by intraseptal injection of the selective antagonist Atosiban impaired social exploration like the effect of Magel2 deficiency (Fig. 2c). Consistently, the blockade of septal AVPR at T1 and OXTR at T3 modified theta rhythmicity throughout the remaining trials (Fig. 2d). Together, social exploration correlated with theta rhythmicity in these mice unless septal AVPR and OXTR were inhibited at T1 (Fig. 2e) and T3 (Fig. 2f), respectively. Therefore, timely actions of AVPR and OXTR in septum generate a sequence of brain activity patterns aligned with the degree of novelty of the social stimulus.

\section{Social salience depends on the AVP and OXT hypothalamoseptal circuits}

To investigate timely activations of AVP and OXT neuronal networks, we first identified which cells responded throughout social trials to target their projections to LS with optogenetic constructs. We extended the initial c-Fos mapping to four brain regions containing AVP and OXT neurons: PVN, SON, BNST and $\mathrm{LH}^{31-33}$. AVP neurons were activated at T1 in the PVN of WT mice and in the BNST of Magel2 ${ }^{+m /-p}$ mice (Fig. S3a,b). OXT neurons were activated at T4 in the PVN of WT mice but less significantly in Magel2 ${ }^{+m /-p}$ mice (Fig. S3c,d). Thus, PVN neurons could be responsible for the release of AVP or OXT in the septum of WT mice unlike the septum of Magel2 $2^{+m /-p}$ mice that could rely on BNST neurons to secrete AVP.

To determine if the aforementioned sources of AVP and OXT modulate social behavior, we adopted an optogenetic silencing strategy. To this end, we used $A v p$-CRE and Oxt-CRE transgenic mice to independently target AAV virus coding for the CRE-dependent halorhodopsin (NpHR3.0-YFP) or eYFP into the PVN or BNST as indicated in Fig. 3a. CREmediated recombination was specific and efficient to express NpHR3.0-YFP either in OXT neurons of PVN or in AVP neurons of PVN or BNST (Fig. 3b). A prerequisite to operate as PVN-LS or BNST-LS circuits responding to social trials was that these neurons projected YFPpositive axon boutons into the septum (Fig. S4a). As expected, yellow light stimulation of recombinant NpHR3.0 in PVN of coronal brain slices reduced the firing rate of target neurons (Fig. 3c). In LS coronal slices, patch clamp recordings of predefined AVP-responding cells or TGOT-responding cells determined the impact of optogenetic manipulations specifically at axon boutons (Fig. S4b). Yellow light stimulation of NpHR3.0 on these cells had no effect in absence of social trials (Fig. S4b-d). On the contrary, blue light stimulation of ChR2-YFP (expressed with similar viral strategy) evoked responses typical of AVP in predefined AVP-responding cells in Avp-CRE animals (Fig. S4e) as well as TGOT in predefined TGOT-responding cells in Oxt-CRE animals (Fig. S4f). Importantly, blue-light evoked responses were blocked by the AVPR antagonist at AVP-responding cells, and OXTR antagonist at TGOT-responding cells. These results validated the optogenetic control of OXT or AVP releases from axon boutons in LS.

Optic fibers were chronically implanted atop LS bilaterally of WT mice to achieve lightdependent silencing of projecting axons from AVP neurons at T1 and from OXT neurons at T3. We found that yellow light stimulation of NpHR3.0 in the PVN-LS AVP pathway (Fig. 3d right) and the PVN-LS OXT pathway (Fig. 3e) impaired social exploration distinctly. 
Hypothalamoseptal pathways activated timely to generate a functional sequence of AVP and OXT septal releases according to the degree of novelty of the social stimulus. In contrast, silencing of an extra-hypothalamoseptal pathway, the BNST-LS pathway failed to modify exploration through social trials (Fig. 3d left), highlighting remarkable specificity about AVP input source to the LS of WT mice under these behavioral conditions.

\section{Disarray of AVP and OXT septal releases disrupted exploration of social salient stimuli}

As sequential septal release of AVP and OXT is critical to express social exploration, we aimed to disrupt this orderly sequence during social trials with optogenetic stimulation of the hypothalamoseptal pathways. We used CRE-dependent ChR2-YFP or eYFP constructs delivered into the PVN or BNST of Avp-CRE and Oxt-CRE mice and induced light stimulation of LS projecting axons from the BNST-LS or PVN-LS circuits to alter the orderly sequence of AVP and OXT releases (Fig. S5a-c). Deficits of social exploration manifested if AVP was released at T3 instead of T1 from BNST-LS pathway and if OXT was secreted at T1 instead of T3 from PVN-LS pathway (Fig. S5d-e). Therefore, it is not the releases of AVP and OXT per se, but its orderly sequence aligned to the degree of social novelty that determined social exploration.

\section{Paucity of cells responding to AVP and OXT orderly sequence in LS of Magel2 $^{+m /-p}$ mice}

Cellular targets in the LS of AVP and OXT orderly sequence remained to be explored. We used patch clamp recordings in coronal brain slices to characterize neurons in LS based on changes of firing rate upon bath application of AVP or TGOT. Half of the cells were selectively excited by AVP (type I) whereas the others were either stimulated selectively by TGOT (type II), or inhibited by both peptides (type III) (Fig. 4a). Retrobead anatomical tracing (Fig. 4b) revealed that the type II and III cells mostly, projected to the medial septum (MS). Connection between this pathway and the hippocampus ${ }^{34}$ is known to organize sequential content of sensory experiences via theta-paced sequence of cell assemblies ${ }^{35-37}$. Modulation of sequential content by LS neurons may rely on inputs containing AVP from the hypothalamus, and glutamate from hippocampus $^{38}$, whereas outputs to MS are enriched with OXTR, suggesting that both peptides could act at different levels of this circuit. Electrophysiological response of the type III cells depended on the orderly sequence of AVP and TGOT contrary to the other cell types recorded. That is, AVP must be presented first to gain responsiveness to TGOT while the effect of AVP was unconditional to the order of presentation (Fig. 4c). These neurons also differed in terms of spontaneous activity patterns, morphology, and other electrophysiological properties (Fig S6).

Importantly, type III neurons were scarcer while type II neurons were denser in Magel2 ${ }^{+m /-p}$ mice than in WT controls (Fig. 4d). Such a change of proportion between cell types could depend on OXTR and AVPR dual expression. To distinguish AVPR and OXTR binding sites with cellular resolution, we synthetized $\mathrm{d}\left[\mathrm{Lys}\left(\right.\right.$ Alexa-Fluor-647) $\left.{ }^{8}\right] \mathrm{VP}$, a fluorescent peptide selective for mouse OXTR in vitro (Fig. 5a) and in vivo if co-injected with the competitive AVPR ligand MC (Fig. 5b bottom left). For a rather selective labeling of mouse AVPR(s) in vivo, higher dose of the fluorescent peptide was used with the competitive OXTR ligand TGOT (Fig. 5b bottom right). When injected in LS after social trials, d[Lys(Alexa-Fluor-647) $\left.{ }^{8}\right]$ VP marked cells equipped with OXTR or AVPR, some of which also contained the activitydependent indicator p-S6 and retrobeads (Fig. 5c). Specifically, LS cells projecting to MS were more abundant and more responsive to social trials in WT controls than in Magel2 ${ }^{+m /-p}$ mice (Fig. 5d,e). These cells are mostly GABAergic somatostatin neurons labelled with retrobeads 
(likely the type III, Fig. S7). All in all, the septum of Magel2 ${ }^{+m /-p}$ mice is ill equipped to organize sequential content of social signals evoked by AVP and OXT releases (Fig. $\mathbf{5 f})$.

\section{AVPR priming in LS of Magel2 $^{+m /-p}$ mice restored exploration of social salient stimuli}

To normalize theta-paced sequence of cell assemblies in the LS of Magel2 ${ }^{+m /-p}$ mice, we promoted AVPR septal response during the first social encounter (Fig. S8d). For this, Magel2 $2^{+m / p}$ mice were cannulated in LS to receive bilateral AVP injections at T1, which increased theta rhythmicity throughout trials (Fig. S8a) and restored social exploration (Fig. 6a). These activities were not correlated if $\mathrm{NaCl}$ or $\mathrm{AVP}+$ Atosiban were injected instead of AVP alone (Fig. 6b and methods for details about in vivo pharmacology). Consistently, $\mathrm{NaCl}$ or AVP+Atosiban injections failed to restore theta rhythmicity and social exploration (Fig. 6a, S8a). Thus, inhibition of septal OXTR with Atosiban despite AVPR priming highlighted the necessity of AVPR and OXTR collective responses to restore social behavior of Magel2 ${ }^{+m /-p}$ mice.

In a second experiment, we promoted the OXT system of Magel2 ${ }^{+m /-p}$ mice given its clinical potential for alleviating social disabilities in humans ${ }^{39,40}$. To this end, we used optogenetic stimulation of CRE-dependent ChR2-YFP recombined in OXT neurons of PVN in Oxt-CRE x Magel2 ${ }^{+m / p}$ mice to promote OXT septal release during social habituation. This manipulation increased exploration duration with known and unknown mice without discrimination (Fig. 6c), and exploration duration correlated with changes of theta rhythmicity (Fig. 6d). Theta rhythm of Magel $2^{+m /-p}$ mice optogenetically-stimulated for OXT septal release (Fig. S8b) looks alike that of WT mice optogenetically-deprived of AVPR septal response at T1 (Fig. 2d). Thus, OXT therapies may not be optimally effective in diseases characterized by AVPR sequential priming defects.

In a third experiment, we used optogenetic stimulation of ChR2-YFP recombined in

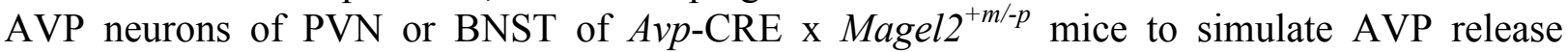
normally evoked by the first social encounter. We found that blue light-stimulation of the BNST-LS AVP pathway promoted consistent social exploration (Fig. 6e right) unlike stimulation of the PVN-LS AVP pathway (Fig. 6e left). Optogenetic stimulation of BNST-LS AVP terminals of Magel2 $2^{+m /-p}$ mice modulated theta rhythmicity (Fig. S8c) that correlated with social exploration (Fig. 6f). This result indicates that social behavior is restored in Magel2 $2^{+m /-p}$ mice by promoting AVPR septal response during the first encounter through the BNST-LS AVP pathway.

Collectively, one promising avenue for disease modification is to restore the orderly sequence of AVP and OXT responses for organizing theta-paced sequence of social salient information flow through the septum.

\section{Discussion}

One major feature of physiopathology associated with Magel2 deficiency reported in this study is the abnormal functional topology of both the AVP and OXT neuronal networks innervating the septum. This is decisive because AVP and OXT must act collectively on septal neurons to demonstrate social salience. Normally, AVP acts first on septal neurons upon release evoked by the novelty of the social stimulus while OXT acts second on septal neurons upon release elicited by the repetition of the social stimulus as previously suggested in rats ${ }^{41,42}$. It is the degree of social novelty that commanded sequential activations of AVP and OXT hypothalamoseptal neuronal networks as seen with the c-Fos mapping studies. 
Pathologically, not only septal AVP fibers were scarcer and PVN AVP neurons poorly activated upon social novelty but also a cryptic extra-hypothalamoseptal AVP network originating from the BNST was activated by social encounters. This suggested that a functional map-to-action relevant for expressing sequential content as described in the hippocampus ${ }^{36}$ could differ between Magel2 $2^{+m /-p}$ mice and WT controls for perceiving the degree of social novelty. Perhaps, new social encounters are seen as threatening more than rewarding by Magel $2^{+m /-p}$ mice, resulting in the mobilization of alternate circuit pathways that influence behavioral response. For instance, threats, unpredictability and social anxiety activate the BNST in which the lesion of AVP cells specifically, reduced social anxiety and aggressiveness ${ }^{43,44}$. Many brain regions among which the PVN and BNST induced $c-F o s$ and either Oxtr or $A v p r(s)$ if conspecific odors came from healthy individuals or sick individuals, respectively. Further blockade of AVPR inhibited social avoidance to sick odors ${ }^{45}$, thus providing evidence that socially evoked activation of AVP BNST cells is an appropriate response to threats that Magel2 $2^{+m /-p}$ mice may privilege even with healthy conspecific. In fact, activation of LSD neurons, a region rich of AVP fibers, inhibits aggressive behavior in mice via its projections to the ventromedial hypothalamus ${ }^{46}$. In Magel2 ${ }^{+m /-p}$ mice, c-Fos activation was elevated in all septal areas under isolation, failing to respond upon social encounters unlike healthy controls, indicating a possible conflict between social perception and theta paced neuronal activation in the social brain network ${ }^{27}$. Despite their social disabilities, Magel2 ${ }^{+m /-p}$ mice perform well with object exploration, exhibiting correlated activities with theta rhythmicity. This contrasts with the WT mice that did not show such correlated activities in the object trials. In agreement, the sensitivity for discriminating faces and objects was reported respectively, impaired and enhanced in adolescents with $\mathrm{ASD}^{47}$ which adds to the face validity of the Magel $2^{+m /-p}$ mice as model of ASD.

Abnormalities in the OXT PVN-LS pathway were less prominent than in the AVP system of Magel2 $2^{+m /-p}$ mice. This is surprising considering the alteration of oxytocinergic neurons function described in Magel $2^{+m /-p}$ mice ${ }^{48}$ but could be due to compensatory mechanisms on post-synaptic target cells in the LSD such as the replacement of type III neurons by the type II or such as the increase of OXT fibers in the LS. Besides the apparent disarray between AVP and OXT septal releases in Magel2 $2^{+m /-p}$ mice, abnormal septal response to social salience hormones corresponded to the underrepresentation of type III neurons belonging to the LS-MS pathway previously described as sequence generator linked with the hippocampus ${ }^{37,49}$. These neurons, which are unconditionally inhibited by AVPR, must gain competence to be inhibited by OXTR thereby operating as detectors of coincidence organizing the orderly sequence of AVP and OXT septal response within a functional map-to-action. Despite the septum of Magel2 ${ }^{+m /-p}$ mice is ill equipped, promoting AVPR septal response during social novelty restored social behavior more efficiently than by stimulation of OXTR septal response during social habituation. Therefore, restoring a complete orderly sequence of AVPR and OXTR septal releases is essential for organizing sequential content of social salience signals. This mechanism could also have implications for treating human pathologies given that activity of the hypothalamoseptal areas was associated with affiliative emotion ${ }^{50}$, and that OXT given intranasally increases the functional connectivity between the septum and other key areas of the social salience and reward circuits ${ }^{51}$.

Therapeutic priming of AVPR septal response for a few minutes at the time of social novelty restored social discrimination more than $1 \mathrm{~h}$ later by a mechanism requiring OXTR septal response in Magel2 ${ }^{+m /-p}$ mice. Molecular and electrophysiological studies provided some clues to understand this effect. First, AVP-deficient Bratteleboro rats centrally administered 
with AVP corrected for several hours the frequency deficit of theta rhythm ${ }^{52}$. Second, AVPR and OXTR responses overlap in the septum where theta paced sequence of cells assemblies projecting to the hippocampus are modulated by OXT and AVP ${ }^{53}$. Third, sequential content of AVP and OXT in septum is suspected to modify theta paced network activity during social encounter. Consistent with an AVPR priming effect in the LS, AVP stimulation of LS neurons was previously shown to condition subsequent excitatory response to glutamatergic inputs from the hippocampus in rats ${ }^{54,55}$. Fourth, cells detecting the coincidence of AVP and OXT sequential content are underrepresented in septum of Magel2 ${ }^{+m /-p}$ mice. They are GABAergic somatostatinpositive cells (type III) projecting to MS likely equipped with both AVPR and OXTR. Unfortunately, this remains an open question, as d[Lys(Alexa-Fluor-647) $\left.{ }^{8}\right]$ VP did not allow for co-labeling of OXTR and AVPR. Lack of AVPR priming during social novelty impaired theta paced information flow through the septum to express social salience by OXTR modulation during habituation. So, therapeutic AVPR priming of Magel2 ${ }^{+m /-p}$ mice should restore septal network activity in response to social encounter. Consistently, intranasal administration of AVP (but not OXT) increased reciprocated collaboration between humans and its associated reactivity in the $\mathrm{LS}^{56}$ even several days beyond treatment ${ }^{57}$.

In terms of clinical perspectives, it is encouraging that priming of septal AVPR can be achieved even by a cryptic source of AVP (e.g. the BNST-LS pathway), further illustrating that circuit defects can be alleviated by loading the septum with AVP at the right time. Moreover, theta paced septal activity and its modulation by social salience hormones is an opportunity to use EEG recordings for predicting social behavior outcomes as demonstrated in this study and others $^{27}$. In humans, EEG abnormalities and epilepsy have been reported in patients with PWS ${ }^{58}$ and $\mathrm{ASD}^{59}$. Few studies reported deficits of social task-related EEG power spectrum changes in ASD patients ${ }^{60,61}$. Here, we provide not only an EEG signature of social disabilities in Magel2 $2^{+m /-p}$ mice but also a blueprint of traces specific for AVPR and OXTR modulations in mouse septum corroborated in rats ${ }^{53}$. Future research will focus on improving an EEG predictive marker of the sensitivity to AVP and OXT in related pathologies and therapies. This is particularly relevant in pathologies such as PWS, SYS and ASD because of the heterogeneity of clinical features and the responses to treatments (e.g. bumetamide ${ }^{62}$ and oxytocin ${ }^{63}$ ).

\section{CONTRIBUTIONS}

A.M.B, M.G.D and F.J designed and verified analytical methods. A.M.B, Y.D, D.D and D.H carried behavior studies. A.M.B, M.G.D carried electrophysiological studies. A.M.B and Y.D carried stereotaxic injections. Cs.T, A.O and M.M synthesized d[Lys(Alexa-Fluor- 647$\left.)^{8}\right]$ VP, characterized in vitro by G.G and in vivo by F.J and Y.D. E.P performed histology. D.D and E.P verified implantations in postmortem brains. A.M.B and D.D analyzed EEG. F.M and P.C provided critical feedback. A.M.B and F.J wrote the manuscript. All authors reviewed and approved the final manuscript. The authors declare no competing financial interests.

\section{ACKNOWLEDGEMENTS}

This work is supported by ANR (M.G.D, F.M), Fondation Lejeune (M.G.D), Fondation pour la recherche médicale (F.J, A.M.B), Centre hospitalier de Montpellier (D.D., P.C.), Montpellier University (A.M.B), and generous support from R. Makineni, R. Tyner, F. Paulsen (M.M.). We thank from IGF in Montpellier, N. Marchi for sharing EEG devices, B. Boussadia for advices on EEG, M. Arango-Lievano for technical strategies, critical reading of the manuscript and M. Tauber (CHU Toulouse) for discussions on PWS.

\section{ABBREVIATIONS}


AAV, Adeno-associated virus; ASD, Autism spectrum disorder; Ato, Atosiban; AVP, ArginineVasopressin; AVPR, AVP receptor; BNST, Bed stria terminalis nucleus; ChR2, Channel rhodopsin-2; EEG, electroencephalogram; GABA, gamma-aminobutyric acid; GLU, glutamate; KO, Knockout; LH, Lateral hypothalamus; LS, Lateral septum; LSD, LS dorsal; LSI, LS intermediate; LSV, LS ventral; Magel2, MAGE family member L2; MC, Manning Compound; MS, Medial septum; NpHR3, Halorhodopsin-3; OXT, Oxytocin; OXTR, OXT receptor; PVN, Paraventricular nucleus; PWS, PraderWilli Syndrome; p-S6, phospho-protein ribosomal S6; SON, Supraoptic nucleus; SYS, Schaaf-Yang syndrome; WT, wildtype; YFP, Yellow protein fluorescent.

\section{REFERENCES}

1. Masi, A., DeMayo, M. M., Glozier, N. \& Guastella, A. J. An Overview of Autism Spectrum Disorder, Heterogeneity and Treatment Options. Neurosci. Bull. 33, 183-193 (2017).

2. Dykens, E. M., Lee, E. \& Roof, E. Prader-Willi syndrome and autism spectrum disorders: an evolving story. $J$. Neurodev. Disord. 3, 225-237 (2011).

3. Schaaf, C. P. et al. Truncating mutations of MAGEL2 cause Prader-Willi phenotypes and autism. Nat. Genet. 45, 1405-1408 (2013).

4. Fountain, M. D. \& Schaaf, C. P. Prader-Willi Syndrome and Schaaf-Yang Syndrome: Neurodevelopmental Diseases Intersecting at the MAGEL2 Gene. Diseases 4, (2016).

5. Kamaludin, A. A. et al. Muscle dysfunction caused by loss of Magel2 in a mouse model of Prader-Willi and Schaaf-Yang syndromes. Hum. Mol. Genet. 25, 3798-3809 (2016).

6. Maillard, J. et al. Loss of Magel2 impairs the development of hypothalamic Anorexigenic circuits. Hum. Mol. Genet. 25, 3208-3215 (2016).

7. Fountain, M. D. et al. The phenotypic spectrum of Schaaf-Yang syndrome - 18 new affected individuals from 14 families. Genet. Med. Off. J. Am. Coll. Med. Genet. 19, 45-52 (2017).

8. Angulo, M. A., Butler, M. G. \& Cataletto, M. E. Prader-Willi syndrome: a review of clinical, genetic, and endocrine findings. J. Endocrinol. Invest. 38, 1249-1263 (2015).

9. Schaller, F. et al. A single postnatal injection of oxytocin rescues the lethal feeding behaviour in mouse newborns deficient for the imprinted Magel2 gene. Hum. Mol. Genet. 19, 4895-4905 (2010).

10. Meziane, H. et al. An Early Postnatal Oxytocin Treatment Prevents Social and Learning Deficits in Adult Mice Deficient for Magel2, a Gene Involved in Prader-Willi Syndrome and Autism. Biol. Psychiatry 78, 8594 (2015).

11. Fountain, M. D., Tao, H., Chen, C.-A., Yin, J. \& Schaaf, C. P. Magel2 knockout mice manifest altered social phenotypes and a deficit in preference for social novelty. Genes Brain Behav. 16, 592-600 (2017).

12. Tauber, M. et al. The Use of Oxytocin to Improve Feeding and Social Skills in Infants With Prader-Willi Syndrome. Pediatrics (2017). doi:10.1542/peds.2016-2976

13. Marlin, B. J. \& Froemke, R. C. Oxytocin modulation of neural circuits for social behavior. Dev. Neurobiol. 77, 169-189 (2017).

14. Shamay-Tsoory, S. G. \& Abu-Akel, A. The Social Salience Hypothesis of Oxytocin. Biol. Psychiatry 79, 194202 (2016).

15. Kendrick, K. M., Guastella, A. J. \& Becker, B. Overview of Human Oxytocin Research. in Behavioral Pharmacology of Neuropeptides: Oxytocin (eds. Hurlemann, R. \& Grinevich, V.) 321-348 (Springer International Publishing, 2018). doi:10.1007/7854_2017_19

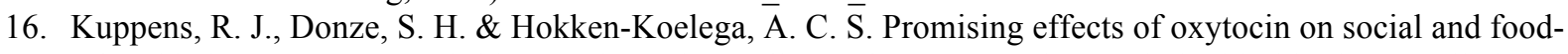
related behavior in young children with Prader-Willi Syndrome: a randomized, double-blind, controlled crossover trial. Clin. Endocrinol. (Oxf.) (2016). doi:10.1111/cen.13169

17. Rice, L. J., Einfeld, S. L., Hu, N. \& Carter, C. S. A review of clinical trials of oxytocin in Prader-Willi syndrome. Curr. Opin. Psychiatry 31, 123-127 (2018).

18. Bales, K. L. et al. Chronic intranasal oxytocin causes long-term impairments in partner preference formation in male prairie voles. Biol. Psychiatry 74, 180-188 (2013).

19. Huang, H. et al. Chronic and Acute Intranasal Oxytocin Produce Divergent Social Effects in Mice. Neuropsychopharmacol. Off. Publ. Am. Coll. Neuropsychopharmacol. (2013). doi:10.1038/npp.2013.310

20. Muscatelli, F., Desarménien, M. G., Matarazzo, V. \& Grinevich, V. Oxytocin Signaling in the Early Life of Mammals: Link to Neurodevelopmental Disorders Associated with ASD. Curr. Top. Behav. Neurosci. 35, 239-268 (2018). 
21. Song, Z. \& Albers, H. E. Cross-talk among oxytocin and arginine-vasopressin receptors: Relevance for basic and clinical studies of the brain and periphery. Front. Neuroendocrinol. (2017). doi:10.1016/j.yfrne.2017.10.004

22. Parker, K. J. et al. A randomized placebo-controlled pilot trial shows that intranasal vasopressin improves social deficits in children with autism. Sci. Transl. Med. eaau7356 (2019). doi:10.1126/scitranslmed.aau7356

23. Bolognani, F. et al. A phase 2 clinical trial of a vasopressin V1a receptor antagonist shows improved adaptive behaviors in men with autism spectrum disorder. Sci. Transl. Med. eaat7838 (2019). doi:10.1126/scitranslmed.aat7838

24. Carter, C. S. The Oxytocin-Vasopressin Pathway in the Context of Love and Fear. Front. Endocrinol. 8, (2017).

25. Veenema, A. H. \& Neumann, I. D. Central vasopressin and oxytocin release: regulation of complex social behaviours. Prog. Brain Res. 170, 261-276 (2008).

26. Korotkova, T. et al. Reconciling the different faces of hippocampal theta: The role of theta oscillations in cognitive, emotional and innate behaviors. Neurosci. Biobehav. Rev. 85, 65-80 (2018).

27. Tendler, A. \& Wagner, S. Different types of theta rhythmicity are induced by social and fearful stimuli in a network associated with social memory. eLife 4, (2015).

28. Arango-Lievano, M. et al. Topographic Reorganization of Cerebrovascular Mural Cells under Seizure Conditions. Cell Rep. 23, 1045-1059 (2018).

29. Landgraf, R. et al. V1 vasopressin receptor antisense oligodeoxynucleotide into septum reduces vasopressin binding, social discrimination abilities, and anxiety-related behavior in rats. J. Neurosci. Off. J. Soc. Neurosci. 15, 4250-4258 (1995).

30. Menon, R. et al. Oxytocin Signaling in the Lateral Septum Prevents Social Fear during Lactation. Curr. Biol. CB 28, 1066-1078.e6 (2018).

31. Grinevich, V., Knobloch-Bollmann, H. S., Eliava, M., Busnelli, M. \& Chini, B. Assembling the Puzzle: Pathways of Oxytocin Signaling in the Brain. Biol. Psychiatry 79, 155-164 (2016).

32. Rood, B. D. \& De Vries, G. J. Vasopressin innervation of the mouse (Mus musculus) brain and spinal cord. $J$. Comp. Neurol. 519, 2434-2474 (2011).

33. Sukhov, R. R., Walker, L. C., Rance, N. E., Price, D. L. \& Young, W. S. Vasopressin and oxytocin gene expression in the human hypothalamus. J. Comp. Neurol. 337, 295-306 (1993).

34. Leranth, C. \& Frotscher, M. Organization of the septal region in the rat brain: cholinergic-GABAergic interconnections and the termination of hippocampo-septal fibers. J. Comp. Neurol. 289, 304-314 (1989).

35. Bender, F. et al. Theta oscillations regulate the speed of locomotion via a hippocampus to lateral septum pathway. Nat. Commun. 6, 8521 (2015).

36. Buzsáki, G. \& Tingley, D. Space and Time: The Hippocampus as a Sequence Generator. Trends Cogn. Sci. 22, 853-869 (2018).

37. Tsanov, M. Differential and complementary roles of medial and lateral septum in the orchestration of limbic oscillations and signal integration. Eur. J. Neurosci. 48, 2783-2794 (2018).

38. Jakab, R. L., Naftolin, F. \& Leranth, C. Convergent vasopressinergic and hippocampal input onto somatospiny neurons of the rat lateral septal area. Neuroscience 40, 413-421 (1991).

39. Benner, S. \& Yamasue, H. Clinical potential of oxytocin in autism spectrum disorder: current issues and future perspectives. Behav. Pharmacol. 29, 1-12 (2018).

40. Kabasakalian, A., Ferretti, C. J. \& Hollander, E. Oxytocin and Prader-Willi Syndrome. Curr. Top. Behav. Neurosci. 35, 529-557 (2018).

41. Lukas, M., Toth, I., Veenema, A. H. \& Neumann, I. D. Oxytocin mediates rodent social memory within the lateral septum and the medial amygdala depending on the relevance of the social stimulus: male juvenile versus female adult conspecifics. Psychoneuroendocrinology 38, 916-926 (2013).

42. Lukas, M., Bredewold, R., Landgraf, R., Neumann, I. D. \& Veenema, A. H. Early life stress impairs social recognition due to a blunted response of vasopressin release within the septum of adult male rats. Psychoneuroendocrinology 36, 843-853 (2011).

43. Clauss, J. A., Avery, S. N., Benningfield, M. M. \& Blackford, J. U. Social anxiety is associated with BNST response to unpredictability. Depress. Anxiety (2019). doi:10.1002/da.22891

44. Rigney, N., Whylings, J., Mieda, M., de Vries, G. J. \& Petrulis, A. Sexually Dimorphic Vasopressin Cells Modulate Social Investigation and Communication in Sex-Specific Ways. eNeuro 6, (2019).

45. Arakawa, H., Arakawa, K. \& Deak, T. Oxytocin and vasopressin in the medial amygdala differentially modulate approach and avoidance behavior toward illness-related social odor. Neuroscience 171, 1141-1151 (2010). 
46. Wong, L. C. et al. Effective Modulation of Male Aggression through Lateral Septum to Medial Hypothalamus Projection. Curr. Biol. CB 26, 593-604 (2016).

47. Pallett, P. M., Cohen, S. J. \& Dobkins, K. R. Face and Object Discrimination in Autism, and Relationship to IQ and Age. J. Autism Dev. Disord. 44, 1039-1054 (2014).

48. Ates, T. et al. Inactivation of Magel2 suppresses oxytocin neurons through synaptic excitation-inhibition imbalance. Neurobiol. Dis. 121, 58-64 (2019).

49. Deng, K. et al. Whole-brain mapping of projection from mouse lateral septal nucleus. Biol. Open 8, bio043554 (2019).

50. Moll, J. et al. A neural signature of affiliative emotion in the human septohypothalamic area. J. Neurosci. Off. J. Soc. Neurosci. 32, 12499-12505 (2012).

51. Rilling, J. K., Chen, X., Chen, X. \& Haroon, E. Intranasal oxytocin modulates neural functional connectivity during human social interaction. Am. J. Primatol. 80, e22740 (2018).

52. Urban, I. J. Effects of vasopressin and related peptides on neurons of the rat lateral septum and ventral hippocampus. Prog. Brain Res. 119, 285-310 (1998).

53. Urban, I. J. Intraseptal administration of vasopressin and oxytocin affects hippocampal electroencephalogram in rats. Exp. Neurol. 74, 131-147 (1981).

54. Urban, I. J. \& De Wied, D. Effect of vasopressin, oxytocin and peptides derived from these hormones on field potential induced in lateral septum of rats by stimulation of the fimbria fornix. Neuropeptides 7, 41-49 (1986).

55. Van den Hooff, P. \& Urban, I. J. Vasopressin facilitates excitatory transmission in slices of the rat dorsolateral septum. Synap. N. Y. N 5, 201-206 (1990).

56. Rilling, J. K. et al. Effects of intranasal oxytocin and vasopressin on cooperative behavior and associated brain activity in men. Psychoneuroendocrinology 37, 447-461 (2012).

57. Rilling, J. K. et al. Arginine Vasopressin Effects on Subjective Judgments and Neural Responses to Same and Other-Sex Faces in Men and Women. Front. Endocrinol. 8, (2017).

58. Verrotti, A., Soldani, C., Laino, D., d'Alonzo, R. \& Grosso, S. Epilepsy in Prader-Willi syndrome: clinical, diagnostic and treatment aspects. World J. Pediatr. WJP 10, 108-113 (2014).

59. El Achkar, C. M. \& Spence, S. J. Clinical characteristics of children and young adults with co-occurring autism spectrum disorder and epilepsy. Epilepsy Behav. EB 47, 183-190 (2015).

60. Oberman, L. M. et al. EEG evidence for mirror neuron dysfunction in autism spectrum disorders. Cogn. Brain Res. 24, 190-198 (2005).

61. Castelhano, J., Tavares, P., Mouga, S., Oliveira, G. \& Castelo-Branco, M. Stimulus dependent neural oscillatory patterns show reliable statistical identification of autism spectrum disorder in a face perceptual decision task. Clin. Neurophysiol. Off. J. Int. Fed. Clin. Neurophysiol. 129, 981-989 (2018).

62. Lemonnier, E. et al. Effects of bumetanide on neurobehavioral function in children and adolescents with autism spectrum disorders. Transl. Psychiatry 7, e1056 (2017).

63. Parker, K. J. et al. Intranasal oxytocin treatment for social deficits and biomarkers of response in children with autism. Proc. Natl. Acad. Sci. U. S. A. 114, 8119-8124 (2017). 


\section{FIGURE LEGENDS}

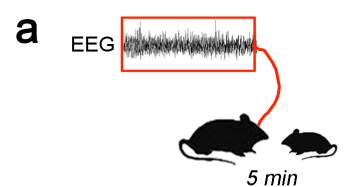

T1: New interaction
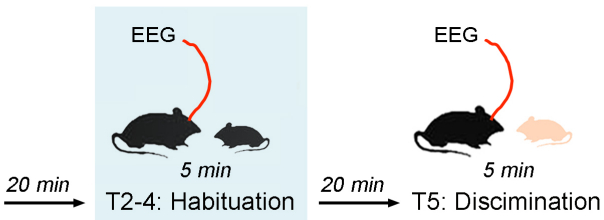

b

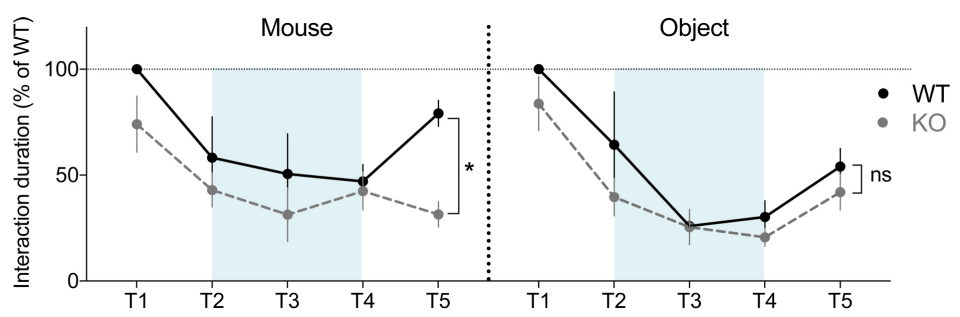

C
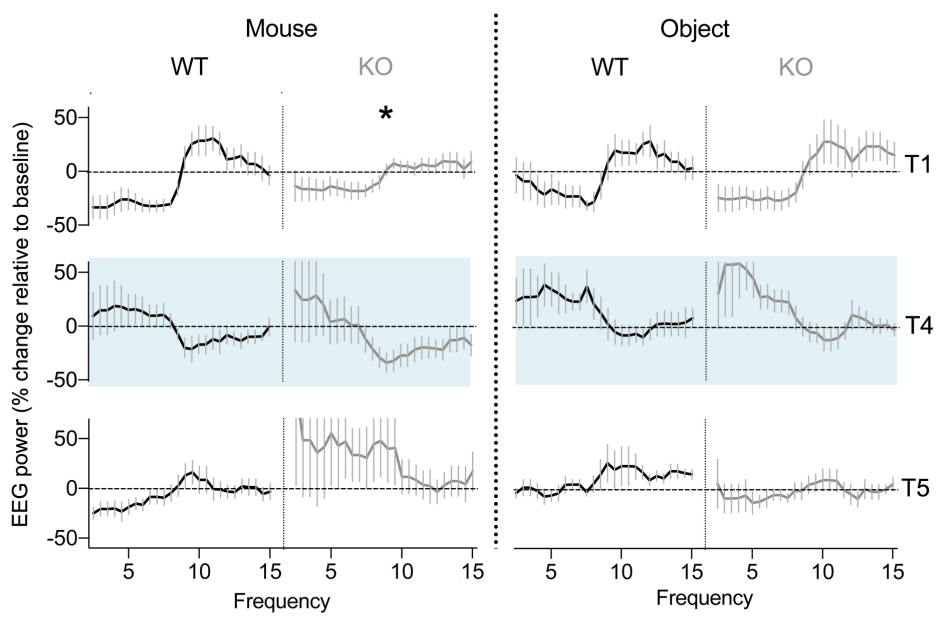

d
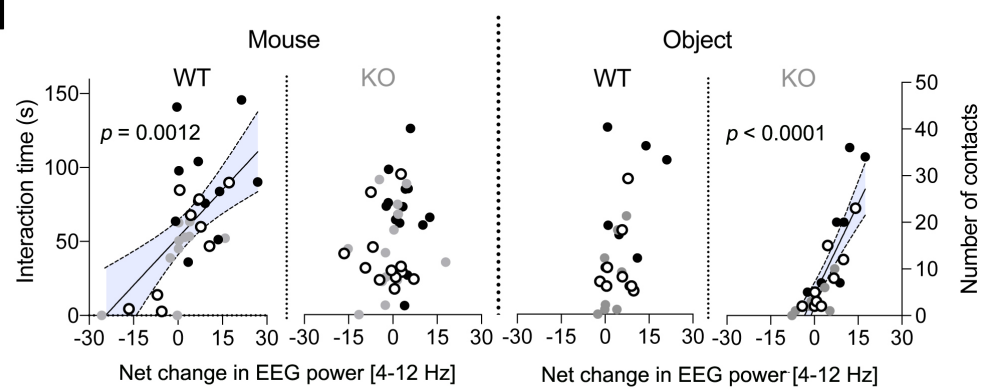

- T1-baseline

- T4-baseline

o T5-baseline

Figure 1. Deficits of EEG theta activity correlated with social defects in $\mathrm{Magel}^{+m /-p}$ mice.

(a) Experimental timeline: EEG recorded during social habituation with a juvenile in 4 successive trials (T1-T4) of 5 min followed by a $5^{\text {th }}$ trial (T5) with a new conspecific.

(b) Time exploring a mouse (left) or an object (right) throughout trials. Data (means \pm SEM) expressed as \% of WT in $\mathrm{n}=15 \mathrm{WT}, 16 \mathrm{KO}$ mice for social and $\mathrm{n}=13 \mathrm{WT}, 15 \mathrm{KO}$ for non-social tests. Two-way ANOVA for social test: effect of trials $p<0.0001$, genotype $p=0.028$, and interaction $p=0.035$, post-hoc Sidak test comparing WT and KO at $\mathrm{T} 5 * p<0.0001$. For non-social test: effect of trials $p<0.0001$, genotype $p=0.14$, and interaction $p=0.71$.

(c) Change in EEG power spectrum during trials. Data (means \pm SEM) expressed as \% relative to baseline in $\mathrm{n}=11 \mathrm{WT}, 13 \mathrm{KO}$ mice for social and $\mathrm{n}=8 \mathrm{WT}, 8 \mathrm{KO}$ for non-social tests. Two-way ANOVA for social test: effect of 
genotype on the theta band at T1 $p<0.0001$, post-hoc Sidak test $*_{p}<0.05$; at T4 $p>0.9$; at T5 $p=0.009$. For nonsocial test: no effect of genotype on the theta band.

(d) Net changes of EEG power in the theta band during trials correlated with behavioral performance. For social test (interaction time in seconds): Spearman coefficient in WT $r=0.56, p=0.0012$ and $\mathrm{KO} r=0.21, p=0.18$. For nonsocial test (number of contact with object): Spearman coefficient in WT $r=0.39, p=0.053$ and KO $r=0.85, p<0.0001$. 
a

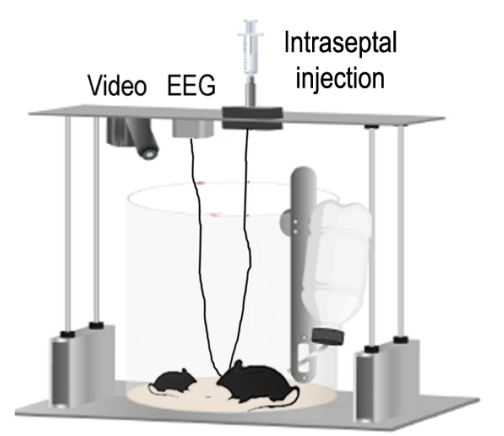

b

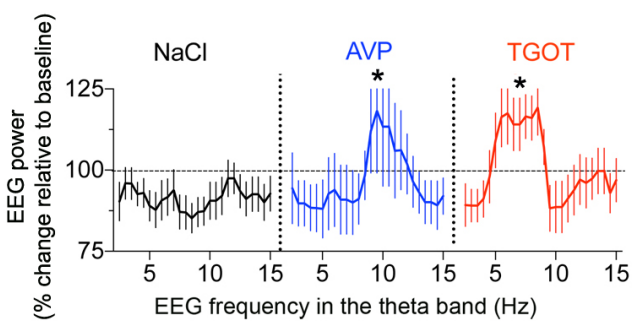

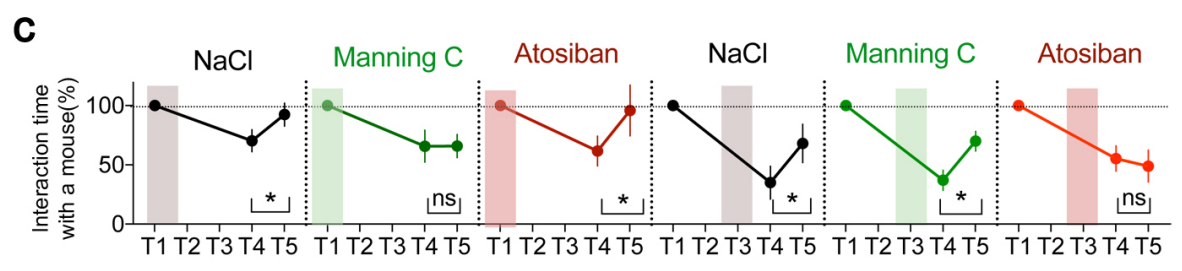

d

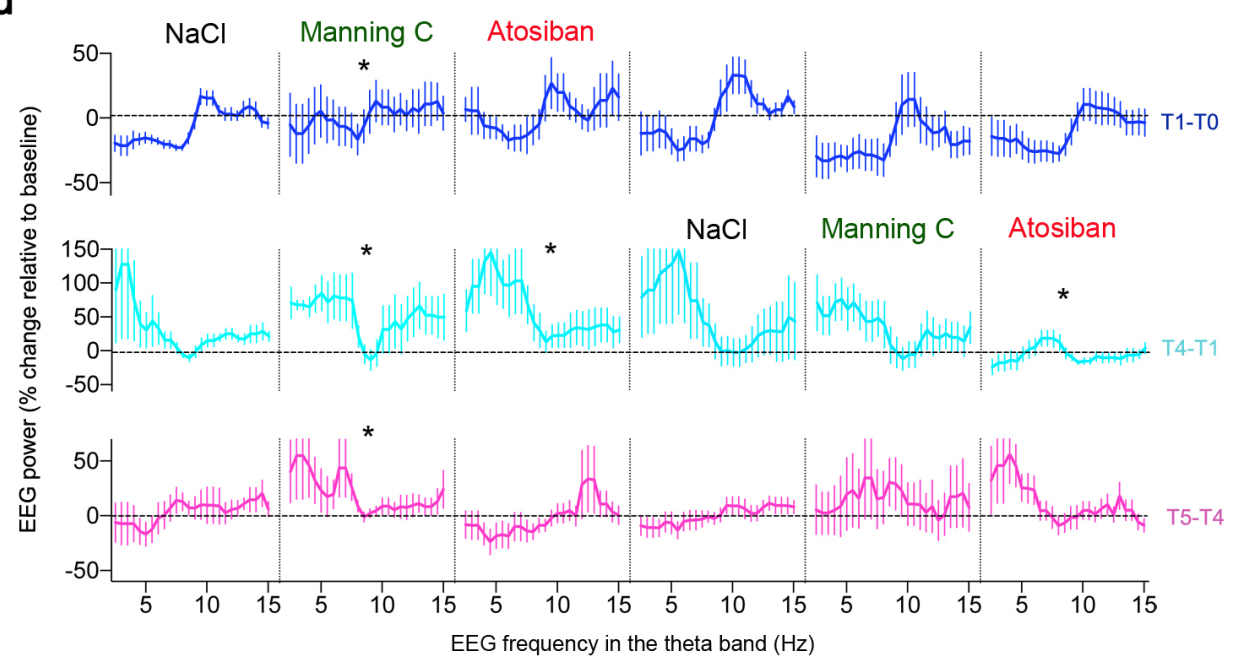

e

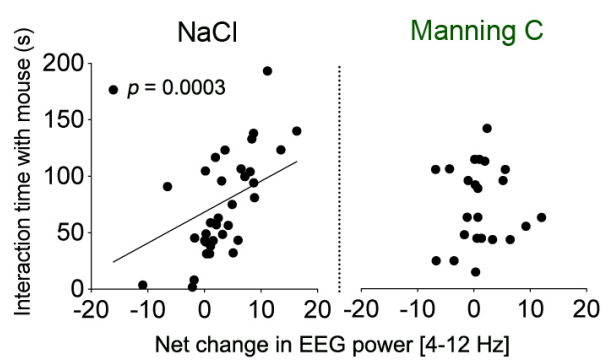

f

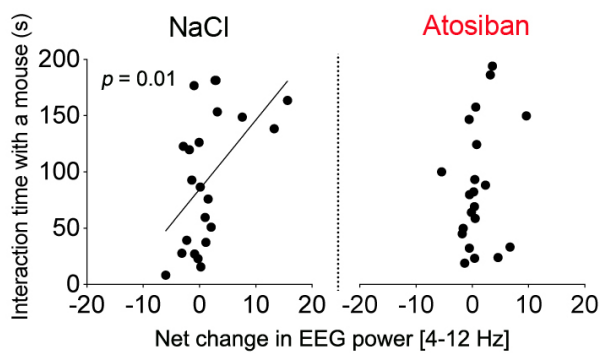

Figure 2. AVP and OXT receptors in septum modulate EEG theta rhythm during social behavior.

(a) Experimental setup to inject drugs in cannulated septum while recording EEG in freely moving mice.

(b) Percent change of EEG power in the low theta band [4-8 Hz] after intraseptal-injection of TGOT $\left(3.10^{-6} \mathrm{M}\right.$ for 9 min) and in the high theta band $[8-12 \mathrm{~Hz}]$ with AVP $\left(3.10^{-5} \mathrm{M}\right.$ for 9 min). Means \pm SEM of $\mathrm{n}=13 \mathrm{NaCl}, 10 \mathrm{AVP}, 10$ TGOT at 10 min post-injection: Kruskal Wallis test $p=0.0011$, effect of AVP ${ }^{*} p=0.04$ and TGOT ${ }^{*} p=0.0005$.

(c) Time exploring a mouse throughout trials upon intra-septal injection of Manning Compound $\left(10^{-8} \mathrm{M}\right)$, atosiban $\left(10^{-8} \mathrm{M}\right)$ or vehicle at $\mathrm{T} 1$ or T3. Data (means $\left.\pm \mathrm{SEM}\right)$ expressed as $\%$ relative to $\mathrm{T} 1 \mathrm{in} \mathrm{n}=16 \mathrm{NaCL}, 9 \mathrm{MC}, 9$ Atosiban 
mice injected at $\mathrm{T} 1$ and $\mathrm{n}=8 \mathrm{NaCL}, 8 \mathrm{MC}, 8$ Atosiban mice injected at T3. Two-way ANOVA: effect of trials $p<0.0001$, post-hoc Dunnett test comparing $\mathrm{T} 4$ and $\mathrm{T} 5 \mathrm{for} \mathrm{NaCl}{ }^{*} p=0.042$, for Atosiban $* p=0.023$. Two-way ANOVA: effect of trials $p<0.0001$, post-hoc Dunnett test comparing T4 and T5 for $\mathrm{NaCl} * p=0.0017$, for $\mathrm{MC}$ $* p=0.0048$. See the methods for the selectivity of MC and Atosiban on mOXTR and mAVPR.

(d) Change of EEG power spectrum at T1 (top), T4 (middle) and T5 (bottom). Effect of intraseptal-injections at T1 of vehicle $(\mathrm{NaCl})$, Manning $\mathrm{C}\left(\mathrm{MC} 10^{-8} \mathrm{M}\right)$ or atosiban (Ato $\left.10^{-8} \mathrm{M}\right)$. Data (means $\pm \mathrm{SEM}$ ) expressed as $\%$ relative to baseline in $\mathrm{n}=11 \mathrm{NaCl}, 8 \mathrm{MC}, 7$ Ato mice: Kruskal Wallis test at $\mathrm{T} 1 p=0.021$, effect of $\mathrm{MC} * p=0.0158$; at T4 $p=0.0015$, effect of $\mathrm{MC} * p=0.0052$ and Ato $* p=0.0026$; at T5 $p<0.0001$, effect of $\mathrm{MC} * p=0.007$. Effect of intraseptal-injections at T3. Means \pm SEM of $\mathrm{N}=8 \mathrm{NaCl}, 7 \mathrm{MC}, 9$ Ato mice: Kruskal Wallis test at $\mathrm{T} 4 p<0.0001$, effect of Ato $* p<0.0001$; at T5 $p=0.0003$, effect of Ato $* p=0.0028$.

(e) Net changes of EEG power in the theta band between trials and baseline correlated with the interaction time with a mouse. Spearman coefficient if $\mathrm{NaCl}$ injected at $\mathrm{T} 1: r=0.56, p=0.0003$; if $\mathrm{MC}$ injected at $\mathrm{T} 1: r=0.03$, $p=0.86$.

(f) Net changes of EEG power in the theta band between trials and baseline correlated with the interaction time with a mouse. Spearman coefficient if $\mathrm{NaCl}$ injected at $\mathrm{T} 3 r=0.53, p=0.01$; if Ato injected at $\mathrm{T} 3 r=0.3, p=0.17$. 
a
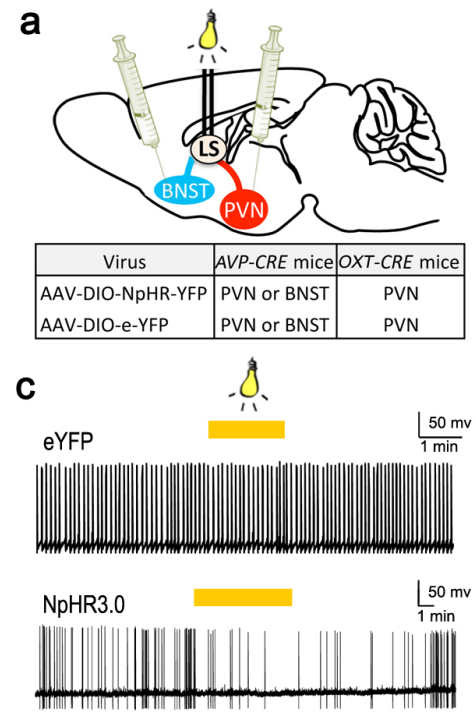

d

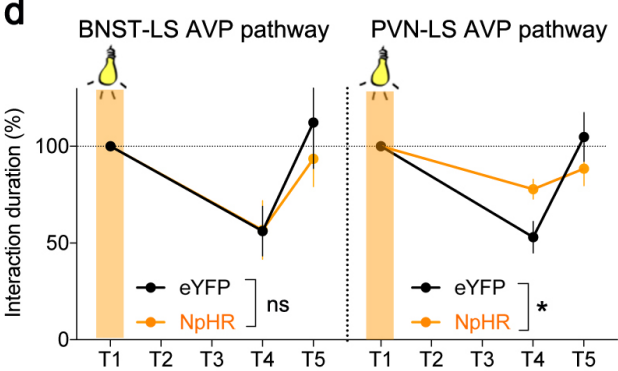

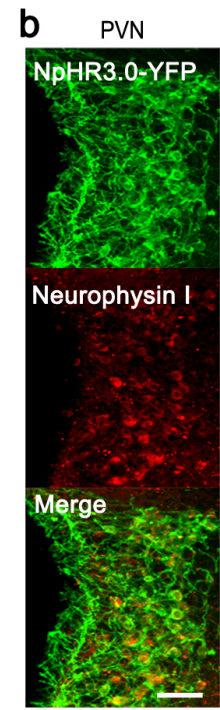
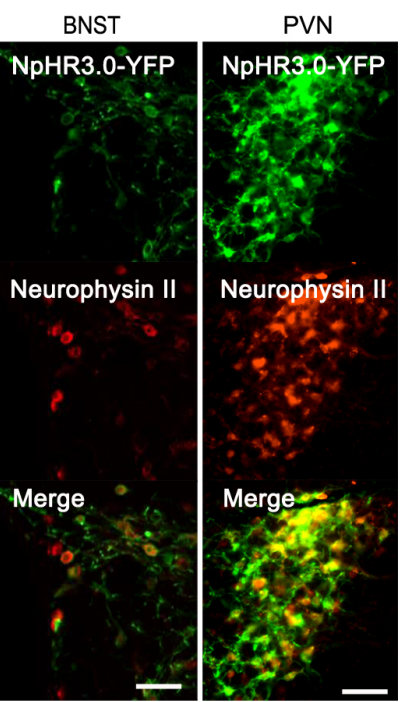

Neurophysin II

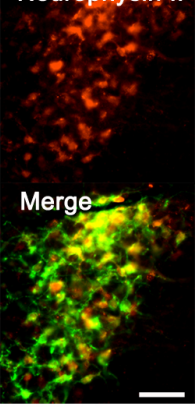

e PVN-LS OXT pathway

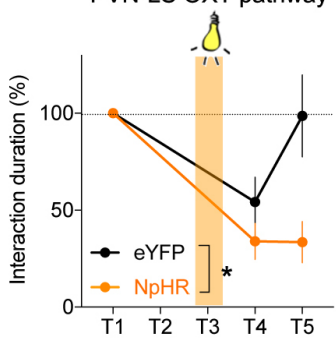

Figure 3. Modulation of social behavior by optogenetic inhibition of the OXT and AVP septohypothalamic circuit pathways.

(a) Viral-mediated optogenetic silencing of septal inputs from OXT neurons (Oxt-CRE mice) in hypothalamic paraventricular nucleus $(\mathrm{PVN})$ or from AVP neurons (Avp-CRE mice) in PVN or Bed nucleus of stria terminalis (BNST).

(b) Co-expression of CRE-mediated NpHR3.0-YFP with Neurophysin I (OXT) in PVN neurons or with Neurophysin II (AVP) in PVN as well as BNST neurons. Scale $=25 \mu \mathrm{m}$.

(c) Firing of action potentials recorded in whole cell configuration in OXT neurons expressing NpHR3.0-YFP or eYFP. Stimulation of cell body with yellow light decreased firing rate.

(d) Time exploring a mouse throughout trials. Data (means \pm SEM) expressed as $\%$ relative to $\mathrm{T} 1$ in each group of n=8 eYFP, 9 NpHR3.0 in BNST and 11 eYFP, 10 NpHR3.0 in PVN of Avp-CRE mice. Two-way ANOVA: Interaction of trials and NpHR3.0 stimulation with light $(561 \mathrm{~nm}$, continuous stimulation, $5 \mathrm{~min}, \sim 2 \mathrm{~mW}$ at $\mathrm{T} 1)$ of BNST fibers: $p=0.6$; of PVN fibers: $p=0.01$ post-hoc Sidak test comparing eYFP with NpHR3.0 at T4 ${ }^{*} p=0.02$.

(e) Time exploring a mouse throughout trials. Data (means \pm SEM) expressed as $\%$ of $\mathrm{T} 1$ in each group of $n=11$ eYFP, 7 NpHR3.0 in PVN of Oxt-CRE mice. Two-way ANOVA: Interaction of NpHR3.0 stimulation of PVN fibers with light $(561 \mathrm{~nm}$, continuous stimulation, $5 \mathrm{~min}, \sim 2 \mathrm{~mW}$ at T3) and trials: $p=0.05$, post-hoc Sidak test comparing eYFP with NpHR3.0 at T5* $p=0.015$. 
a

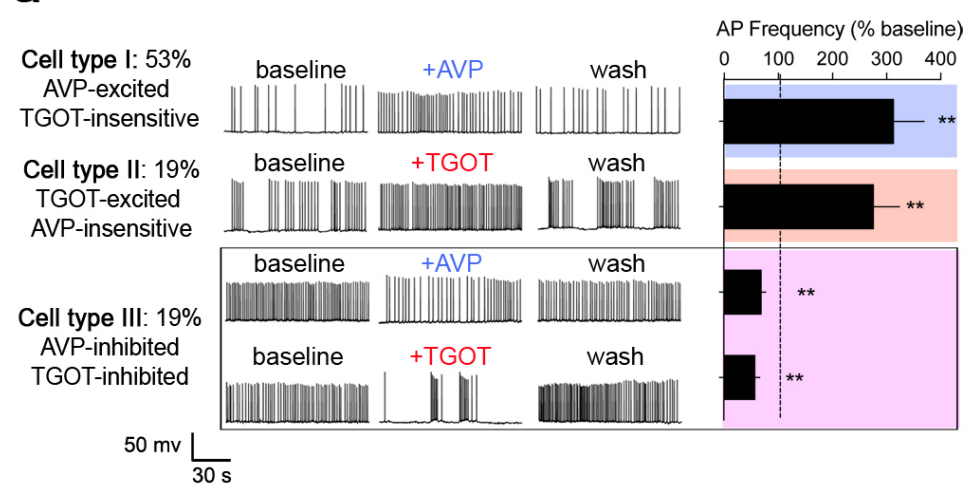

C

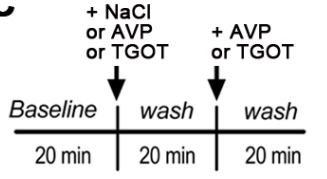

Alternate sequences tested:

(1) NaCl-wash-TGOT-wash

(2) AVP-wash-TGOT-wash

(3) $\mathrm{NaCl}$-wash-AVP-wash

(4) TGOT-wash-AVP-wash

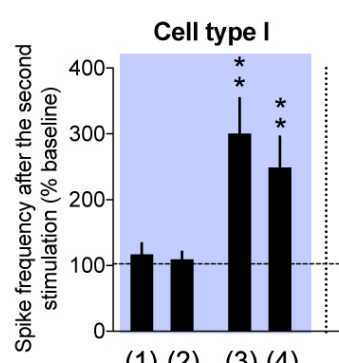

(1) (2) (3) (4)
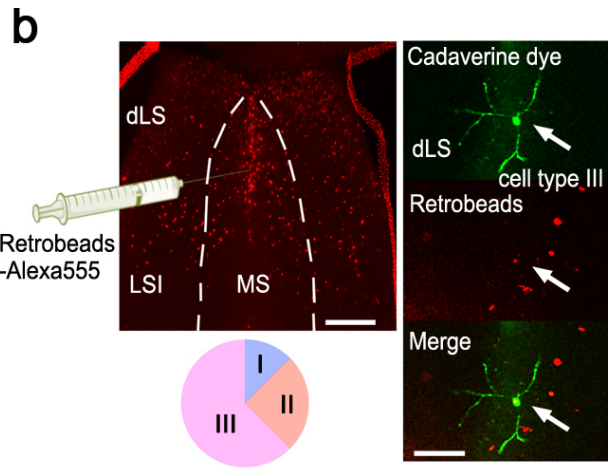

d

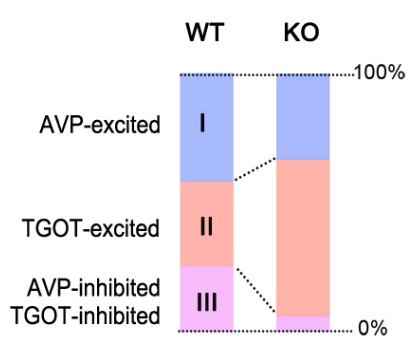

Figure 4. Paucity of cells responding to the orderly sequence of AVP and OXT in LS of Magel2 ${ }^{+m /-p}$ mice

(a) Firing rate of action potentials recorded in whole cell configuration in septal slices at baseline and upon 2-min bath application of AVP $\left(10^{-6} \mathrm{M}\right)$ and TGOT $\left(10^{-7} \mathrm{M}\right)$. All 3 types of response reverted to baseline after washout. Data (means \pm SEM) expressed as \% relative to baseline prior treatment in $\mathrm{n}=29$ TGOT-excited, 34 AVP-excited, 20 AVP-inhibited and 16 TGOT-inhibited cells. T-Test for the effect of TGOT-excitation ** $p=0.0007$; AVP-excitation ** $p=0.0005$; TGOT-inhibition $* * p<0.0001$; AVP-inhibition $* * p=0.001$.

(b) Cells in LSD marked with cadaverine-Alexa-Fluor-594 via the patch pipette corresponded mostly to the type III if they uptake fluorescent retrobeads injected in MS ( $\mathrm{n}=16$ neurons, 2 type I, 4 types II, 10 type III in 11 mice). Scales $=200$ and $50 \mu \mathrm{m}$.

(c) Effect of orderly sequence of AVP and TGOT on spike frequency. Sequence of 2 stimuli (2 min each) to categorize cell types a posteriori. Wilcoxon test for the effect of TGOT alone on type II cells ${ }^{* *} p=0.0027$; AVP $1^{\text {st }}$ - TGOT $2^{\text {nd }}$ on type II cells $*=0.0155$; AVP $1^{\text {st }}-$ TGOT $2^{\text {nd }}$ on type III cells $* * * p<0.0001$; AVP alone on type I cells $* * p=0.0018$; TGOT $1^{\text {st }}-$ AVP $2^{\text {nd }}$ on type I cells $* * p=0.0055$; AVP alone on type III cells $* p=0.039$; TGOT $1^{\text {st }}$ AVP $2^{\text {nd }}$ on type I cells * $p=0.0138$.

(d) Proportion of cells categorized as a function of their responses to AVP and TGOT on the frequency of action potentials. $\mathrm{n}=28 \mathrm{WT}, 11 \mathrm{KO}$ type I cells, $22 \mathrm{WT}, 20 \mathrm{KO}$ type II cells and $17 \mathrm{WT}, 2 \mathrm{KO}$ type III cells in septal slices of 11 Magel2 $^{+m / p}$ and 29 WT controls mice. 

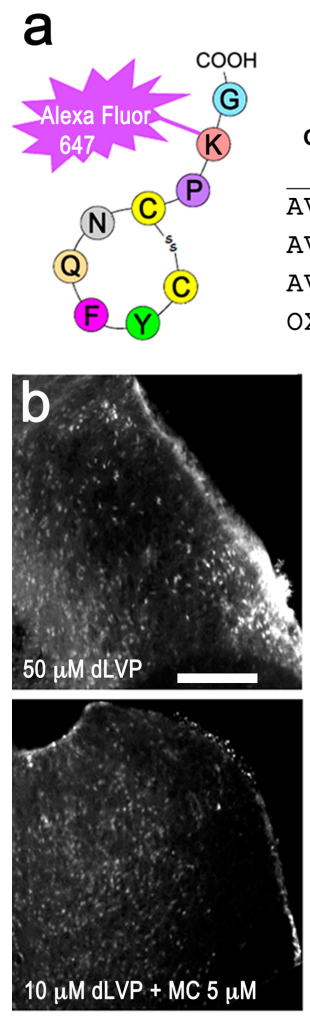

d

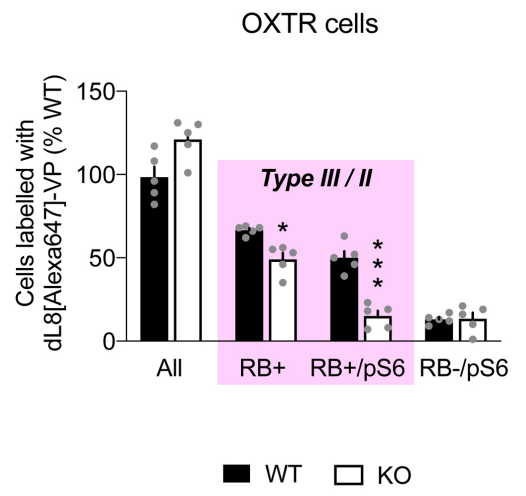

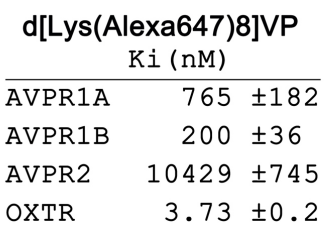

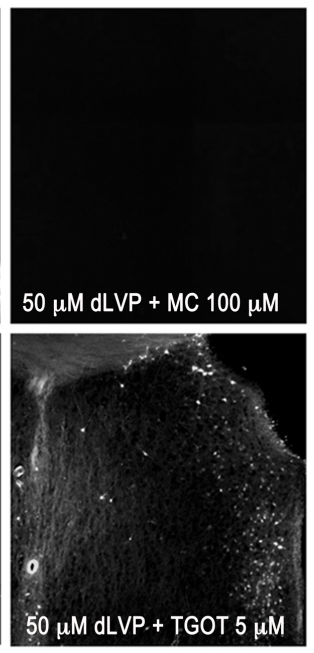

e
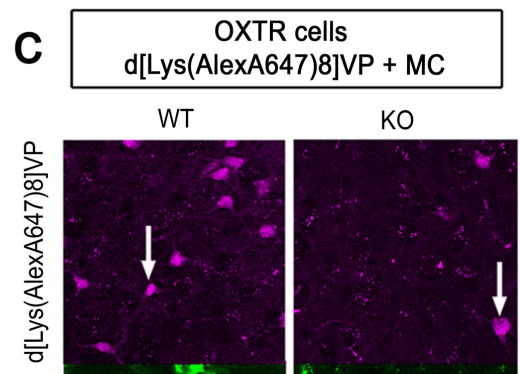

WT

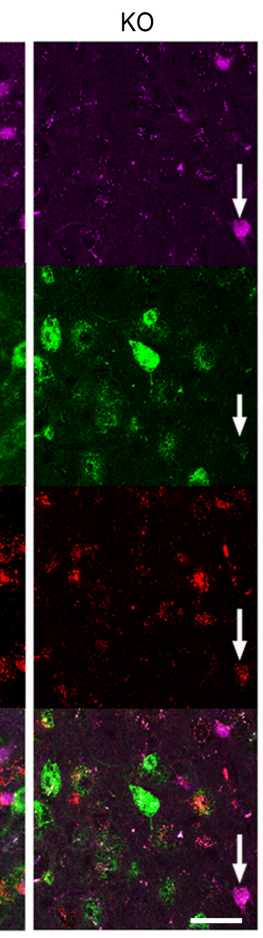

f

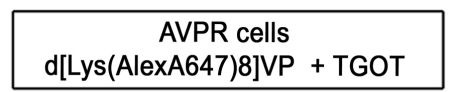

WT KO
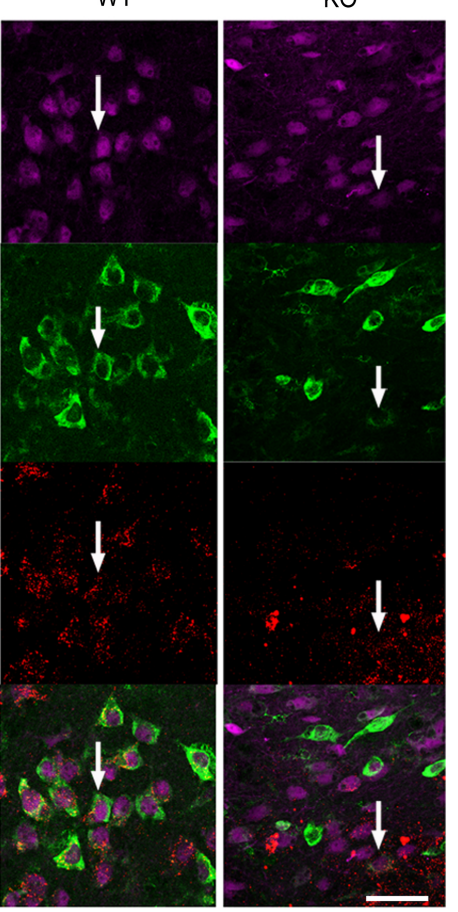

AVPR cells

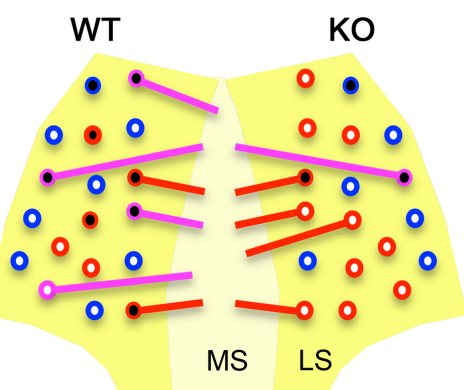

- Type I cells AVP-excited ("Bright"/GLU) - Type II cells OXT-excited (GABA)

- Type III cells AVP/OXT-inhibited ("Dim"/GABA) - $\mathrm{p}$-S6 cells upon social trials at T5

Figure 5. A new fluorescent ligand to identify OXT and AVP responding cells in vivo.

(a) Affinity of $\mathrm{d}\left[\right.$ Lys(Alexa-Fluor-647) $\left.{ }^{8}\right] \mathrm{VP}$ in vitro for the indicated mouse receptors: AVPR1b and OXTR transfected in HEK293 cells and AVPR1a and AVPR2 endogenous from liver and kidney, respectively. Means \pm SEM of $n=3$ independent competition assays against $\left[{ }^{3} \mathrm{H}\right] \mathrm{AVP}$.

(b) Representative in vivo labeling of cells with intraseptal injection of d[Lys(Alexa-Fluor-647) $\left.{ }^{8}\right] \mathrm{VP}$ in control

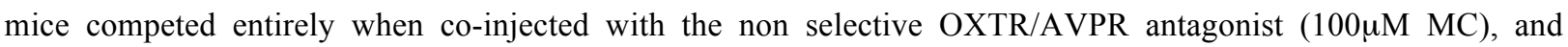
partially with $5 \mu \mathrm{M}$ MC (to block AVPR sites only) or with $5 \mu \mathrm{M}$ TGOT (to block OXTR sites only). Scale $=200 \mu \mathrm{m}$. See the methods for the selectivity of MC and TGOT on mOXTR and mAVPR.

(c) Representative in vivo labeling of OXTR (left) and AVPR (right) binding sites in LS of WT and Magel2 ${ }^{+m /-p}$ mice prior injected with retrobeads (RB-Alexa-Fluor-555) in MS to mark cell type III. Cannulated animals were administered with drugs in LS $5 \mathrm{~min}$ after the last trial of social habitation/discrimination and 10min later, sacrificed. Scale bars $=25 \mu \mathrm{m}$. 
(d) Percent of cells expressing OXTR binding sites and p-S6 in LS. Total OXTR cells 353WT of which 237RB+, $451 \mathrm{KO}$ of which $224 \mathrm{RB}+$. Means \pm SEM of $\mathrm{n}=5 \mathrm{WT}$ and 5 Magel2 $^{+m / p}$ mice. Two-way ANOVA: Effect of genotype on OXTR cells: $p<0.0001$, post-hoc Sidak test for the percent of OXTR cells projecting to MS * $p=0.0415$ and OXTR cells projecting to MS with $\mathrm{pS6} * * * p=0.0002$.

(e) Percent of cells expressing AVPR binding sites and p-S6 in LS. Total AVPR cells 474WT of which 235RB+, $379 \mathrm{KO}$ of which $112 \mathrm{RB}+$. Means \pm SEM of $\mathrm{n}=5 \mathrm{WT}$ and 5 Magel2 $^{+m /-p}$ mice. Two-way ANOVA: Effect of genotype on AVPR cells: $p=0.0256$, post-hoc Sidak test for the percent of AVPR cells projecting to MS * $p=0.011$.

(f) Summary of cell types equipped with AVPR/ OXTR in LS. Type III cells inhibited by both AVP and TGOT are less solicited (p-S6 signaling) in Magel2 ${ }^{+m / p}$ mice compared to WT controls during social trials. 
a

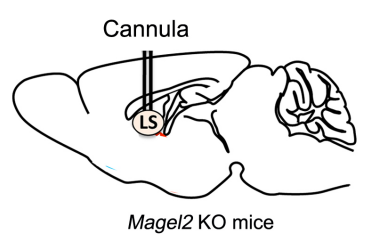

C

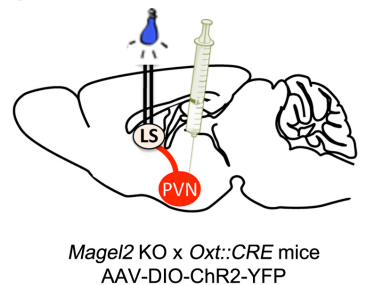
AAV-DIO-ChR2-YFP

e

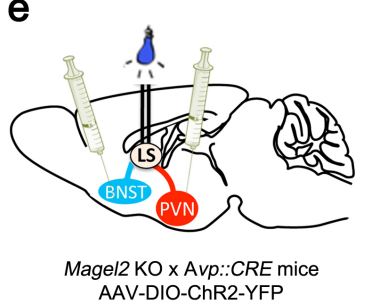

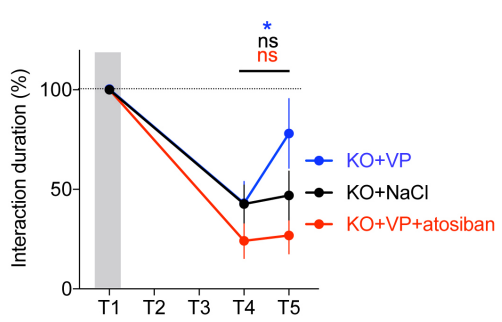

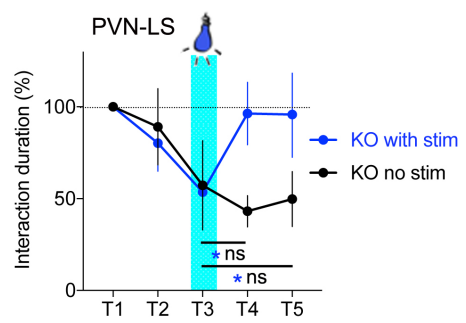

b

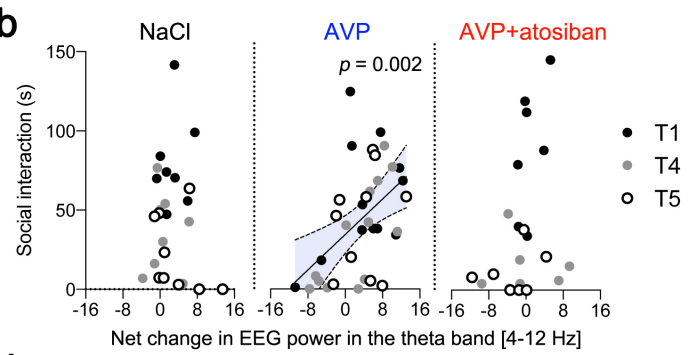

d

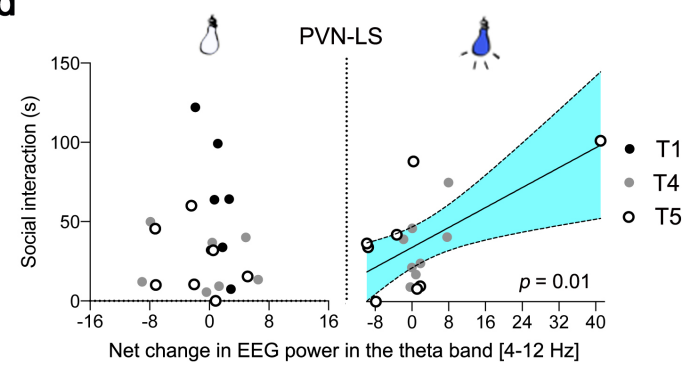

f
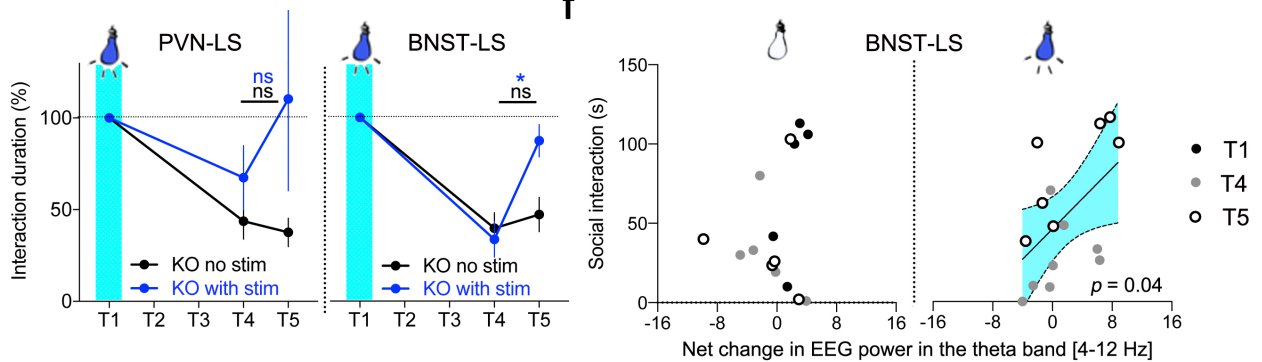

Figure 6. Stimulation of the BNST-LS AVP pathway and PVN-LS OXT pathway restored social behavior in Magel2 $^{+m / p}$ mice.

(a) Time Magel2 $2^{+m / p}$ mice explored WT conspecifics after intraseptal injection of AVP $\left(3.10^{-6} \mathrm{M}\right)$ with or without atosiban $\left(5.10^{-8} \mathrm{M}\right)$ at $\mathrm{T} 1$. Data (means $\pm \mathrm{SEM}$ ) expressed as $\%$ relative to $\mathrm{T} 1$ in each group of $\mathrm{n}=13 \mathrm{NaCl}, 12 \mathrm{AVP}$ and 6AVP+atosiban mice. Two-way ANOVA: Effect of social trials: $p<0.0001$ post-hoc Dunnett test for the effect of NaCl: T4 vs T1 $* p<0.0001$; effect of AVP: T4 vs T1 ${ }^{*} p<0.0001$ and T4 vs T5 $* p=0.01$; effect of AVP+atosiban: $\mathrm{T} 4$ vs $\mathrm{T} 1 * p<0.0001$.

(b) Net changes of EEG power in the theta band between trials correlated with time of social interaction. Pearson coefficient if $\mathrm{NaCl}$ injected at T1: $r=-0.08, p=0.6$; if AVP injected at T1: $r=0.49,{ }^{*} p=0.024$; if AVP+atosiban injected at $\mathrm{T} 1: r=0.3, p=0.18$.

(c) Time Magel2 $2^{+m / p}$ mice explored WT conspecifics after blue light stimulation $(473 \mathrm{~nm}, 30 \mathrm{~Hz}, 10 \mathrm{~ms}$ pulses for 2min, 2mW) of OXT neuron projections between PVN and LS (PVN-LS pathway). Data (means \pm SEM) expressed as $\%$ relative to $\mathrm{T} 1$ in each group of $\mathrm{n}=12$ no stim, 12 stim mice. Two-way ANOVA: Effect of ChR2 stim on social trials: $p=0.0037$ post-hoc Dunnett test for the effect of no stim: T3 vs T1 ${ }^{*} p=0.035$; effect of ChR 2 stim: T3 vs T1 ${ }^{*} p=0.016 \mathrm{~T} 3$ vs T4 $* p=0.029$ and $\mathrm{T} 3$ vs T5 $* p=0.0318$.

(d) Net changes of EEG power in the theta band between trials correlated with time of social interaction after optogenetic stimulation of PVN-LS OXT terminals. Pearson coefficient if no stim at T3: $r=-0.2, p=0.47$; if ChR2 stim at T3: $r=0.62,{ }^{*} p=0.01$.

(e) Time Magel2 $2^{+m-p}$ mice explored WT conspecifics after blue light stimulation $(473 \mathrm{~nm}, 20 \mathrm{~Hz}, 5 \mathrm{~ms}$ pulses for 2min, 2mW) of AVP neuron projections between BNST and LS (BNST-LS pathway) or PVN and LS (PVN-LS pathway). Data (means \pm SEM) expressed as \% relative to $\mathrm{T} 1$ in each group of $\mathrm{n}=9$ no stim, 6 stim BNST-LS mice and 9 no stim, 9 stim PVN-LS mice. (Left) Two-way ANOVA: Effect of ChR2 in PVN-LS on social trials: $p=0.2$ post-hoc Dunnett test for the effect of no stim: T4 vs T1 ${ }^{*} p=0.0008$ and T4 vs T5 $p=0.75$; effect of stim: T4 vs T1 $p=0.16$ and T4 vs T5 $p=0.6$. (Right) Two-way ANOVA: Effect of ChR2 in BSNT-LS on social trials: $p=0.005$ post- 
hoc Dunnett test for the effect of no stim: T4 vs T1 ${ }^{*} p<0.0001$ and T4 vs T5 $p=0.6$; effect of stim: T4 vs T1 ${ }^{*} p<0.0001$ and $\mathrm{T} 4$ vs $\mathrm{T} 5{ }^{*} p=0.01$.

(f) Net changes of EEG power in the theta band between trials correlated with time of social interaction. Pearson coefficient if no stim at T1: $r=-0.17, p=0.07$; if ChR2 stim at T1: $r=0.53,{ }^{*} p=0.04$. 


\section{Methods}

All experiments were carried out in accordance with the Directive by the Council of the European Communities (86/609/EEC) following guidelines from the French Ministry of research and ethics committee for the care and use of laboratory animals (approved protocol APAFIS 5133, 8940, 11468).

\section{Reagents}

Vasopressin, TGOT, Atosiban, Manning Compound, TTX, CGP35348, GABAzine and salts for electrophysiology are from Sigma Aldrich (S.A.R.L, Saint-Quentin, France). Antibodies: mouse monoclonal Neurogranin (Ab5620), NeuN, GAD67, Calretinin (Merck Millipore, St Quentin en Yvelines, France), Calbindin (Swant, Marly, SWI), Guinea Pig polyclonal Neurotensin (Synaptic systems), rat polyclonal Somatostatin, chicken polyclonal GFP (Abcam, Cambridge, UK), rabbit polyclonal c-Fos, p-S6 (Cell Signaling Technology, Ozyme France) and custommade rabbit polyclonal antibodies against neurophysin I and II (Kindly provided by H. Gainer at NIH, Bethesda, USA).

\section{Animals}

Oxttm1.1(CRE)Dolsn/J (Oxt-CRE) and Avptm1.1(cre)Hze/J (Avp-CRE) male mice were purchased (Jackson labs, Bar Harbor, MA, USA) and maintained on a C57B6/J background (Janvier Labs, Le Genest-Saint-Isle, France). Magel2-deficient mice were provided by F. Muscatelli (INMED, Marseille, France) and backcrossed with Oxt-CRE and Avp-CRE lines on a C57B6/J background. Magel $2^{+\mathrm{m} / \mathrm{p}}$ (paternal KO allele) mice were obtained by crossing WT females with ${ }^{+\mathrm{p} / \mathrm{m}}$ (maternal KO allele) males. Magel2 being a maternally imprinted gene, Magel2 ${ }^{+\mathrm{m} / \mathrm{p}}$ mice did not express Magel2 and were considered functionally KO (Magel2 KO) as previously described ${ }^{1}$. Littermates expressing 2 wild type alleles were used as controls. Genotyping primers and protocols are as recommended by the manufacturer. For the Magel 2 WT allele: 5'-GTCACACACCCATTCGACCT-3' and 5'-TACCCTCGGGAGCAGTAGAC-3'; Magel2 KO allele: 5'-TGCTTCCTGCCCTTCAGTTAC-3' and 5'GCTTATCGATACCGTCGACCTC-3'; Cre-recombinase: 5'-TCTGTCCGTTTGCCGGTCGT3' and 5'-AGACCGCGCGCCTGAAGATA-3'; Avp-CRE WT allele : 5'GAGTCCGTGGATTCTGCCAA-3' and 5'-CTATGCACGACTTCGGGTGT-3'; Oxt-CRE WT allele: 5'-CTCAGAACACTGACCCATTTCTCTT-3' CCGACAATTAGACACCAGTCAAG-3'. All animals were housed under a 12/12 light/dark cycle in a SOPF sanitary status with access to ad libitum food and water. Enrichment consisted of cotton pieces to build a nest. Animals were group-caged until surgeries. After implantation surgery, animals were isolated until the end of the experiments. All efforts were made to minimize animal suffering and to reduce the number of mice utilized in each series of experiments.

\section{Behavior}

The effects of Magel2 $2^{+\mathrm{m} / \mathrm{p}}$ mutation were evaluated on behaviors. A week before the $1^{\text {st }}$ experiment, mice (3-4 months old) were habituated to manipulation by the experimenter. A plexiglass transparent circular arena ( $24 \mathrm{~cm}$ diameter) was video-recorded for the time of the experiment and accommodated with clean litter, a water bottle and food before the beginning of the experiment. For social behavior, a transparent box with sniff holes was also introduced before the beginning of the experiment and a test mouse was left for $10 \mathrm{~min}$ to habituate to the arena. After 20 min of basal activity, a juvenile conspecific was introduced in the box for 5 min. 
The same presentation happened 4 times at 20 min intervals to habituate the mice with one another. After another $20 \mathrm{~min}$, a new juvenile different from the first one was introduced in the box for 5 min. The set-up was systematically cleaned using disinfectant swipes before a new experimental mouse was challenged. This test in rodents typically decreases exploration of the known juvenile while it increases exploration of the new juvenile ${ }^{2}$. For non-social behavior, a similar protocol was used except that an object was inserted into the arena. Time spent sniffing a juvenile freely moving or number of interactions with an object was extracted manually and expressed as $\%$ of interaction time during the first encounter.

\section{In vivo pharmacology}

Bilateral cannula (Phymed, 26G) heading to the lateral septum were implanted in mice using a stereotaxic frame (AP $+0.05 \mathrm{~cm}, \mathrm{ML}+/-0.04 \mathrm{~cm}, \mathrm{DV}-0.3 \mathrm{~cm})$ under ketamine $(6.6 \mathrm{~g} / \mathrm{kg})$ xylazine $(1.3 \mathrm{~g} / \mathrm{kg})$ anesthesia. Dental cement (Paladur, Henry Schein) and sutures were used to secure the implant. Animals were given anti-inflammatory medication (doliprane, $6 \mathrm{mg}$ ) and were monitored every day and allowed recovery for 7 days post-surgery. Positions of cannulas were verified a posteriori (Fig. S9a). Drugs were injected through the cannula using bilateral injector connected through 2 different tubing to two $1 \mu \mathrm{L}$ Hamilton seringes controlled by a microinjector pumps (micro 4, World Precision Instrument). Injections of $900 \mu \mathrm{L} / \mathrm{hemisphere}$ were performed at $100 \mathrm{~nL} / \mathrm{min}$ and diffusion perimeter was estimated by injection of Alexa-594cadaverine (Life Technology, $50 \mu \mathrm{M}$, molecular weight comparable to the peptides used) using the same parameters just before euthanasia. The injection set-up was connected to the cannula 5 min before the injection and left for 5 min after the end of the injection such that it overlaps with the interaction with a mouse or object. The same mice were allowed at least 5 days between consecutive injections of $\mathrm{NaCl} 0.9 \%$, Atosiban $\left(10^{-8} \mathrm{M}\right.$ in all experiments except $5.10^{-8} \mathrm{M}$ for fig 6a and $\mathbf{6 b}$ to disrupt OXTR during habituation as 2 consecutive injections at T1 and T3 impaired the robustness of the test), Manning compound $\left(10^{-8} \mathrm{M}\right)$, AVP $\left(3 \times 10^{-6} \mathrm{M}\right)$ and TGOT $\left(3 \times 10^{-7} \mathrm{M}\right)$ performed in random orders ${ }^{3,4}$. Concentrations of aforementioned selective ligands were determined based on published selectivity profiles on mouse receptors ${ }^{5}$. In brief, Atosiban is $>700$ times more potent on mOXTR than mAVPRs; TGOT is $>20,000$ times more potent on OXTR than mAVPRs; MC has a 24-42 times higher affinity for mAVPR1A than for mOXTR and mAVPR1B, respectively. Though AVP binds equally to mOXTR and MAVPR in vitro ${ }^{5}$, it failed to elicit an electrophysiological response on OXT-excited type II neurons of brain slices. Thus, the priming effect of AVP on OXT response of inhibited-type III neurons is unlikely due to desensitization of OXTR.

\section{Optogenetics}

Oxt-CRE, Avp-CRE, Oxt-CRE;Magel2 $2^{+\mathrm{m} /-\mathrm{p}}(\mathrm{KO})$ and Avp-CRE;Magel2 $2^{+\mathrm{m} /-\mathrm{p}}(\mathrm{KO})$ animals were used in these experiments. At 4 weeks, animal were injected with one of the following AAV1 (EF1a::DIO-eNpHR3.0-eYFP;WPRE::hGH; EF1a::DIO-ChR2-eYFP;WPRE::hGH or EF1a::DIO-eYFP;WPRE::hGH from viral vector core, University of Pennsylvania) (500 $\mathrm{nL} /$ hemisphere, $2 \times 10^{11}$ viruses $/ \mathrm{mL}$ ) either in the hypothalamic paraventricular nucleus (AP -0.01 $\mathrm{cm}, \mathrm{ML}+/-0.02 \mathrm{~cm}$, DV $0.48 \mathrm{~cm}$ ) or in the bed nucleus of the stria terminalis (BNST: AP 0.05 $\mathrm{cm}, \mathrm{ML}+/-0.1 \mathrm{~cm}, \mathrm{DV}-0.4 \mathrm{~cm}$ ) using a microinjector-controlled (micro-4, WPI) seringe (nanofil seringe and Nanofil 33G BVLD Needle, WPI). One month after the infection, bilateral optic fibers (dual fiberoptic cannula, Doric lenses, $0.53 \mathrm{NA}$ ) were implanted in the septum (AP $+0.05 \mathrm{~cm}, \mathrm{ML}+/-0.04 \mathrm{~cm}, \mathrm{DV}-0.3 \mathrm{~cm})$. All surgeries were performed under ketamine $(6.6$ $\mathrm{g} / \mathrm{kg})$ xylazine $(1.3 \mathrm{~g} / \mathrm{kg})$ anesthesia. LED were calibrated at $500 \mathrm{~mA}$ to deliver $2 \mathrm{~mW}$ at the tip 
of the optic fiber, as monitored with a luminometer (Thorlab). Stimulations consisted of 5 min continuous stimulation at $561 \mathrm{~nm}$ for the halorhodopsin and $5 \mathrm{~ms}$ pulses, $20 \mathrm{~Hz}$ or $10 \mathrm{~ms}$ pulses, $30 \mathrm{~Hz}$ at $473 \mathrm{~nm}$ for the channelrhodopsin (respectively for vasopressinergic or oxytocinergic neurons stimulation) as previously used ${ }^{6,7}$. Positions of optic fibers were verified a posteriori (Fig. S9b,c).

\section{EEG recordings}

Mice at least 2 months of age were implanted with surface electrodes atop the cortex and allowed two weeks of recovery from surgery before use for experiments. Electroencephalogram (EEG) activity was monitored for $3 \mathrm{~h}$ during interactions with a mouse or an object (Pinnacle Inc., Sarasota, FL, USA). EEG signals were acquired at $600 \mathrm{~Hz}$, stored and analyzed using Sirenia (Pinnacle Inc., Sarasota, FL, USA). Dual Video-EEG inspection permitted the exclusion of artifacts associated with scratching, eating, drinking, chewing, self-grooming, and sleep from interesting period of behavioral interactions with a conspecific or an object. Frequencies above $100 \mathrm{~Hz}$ were filtered and data analyzed with Neuroscore software (Data Science International). Frequency bands of $0.5 \mathrm{~Hz}$ were defined and power of each of these bands was evaluated for each 10s epoch composing the signal. Mean values of power for each band of frequency were calculated for the periods relevant with the behavior (while the social stimulus or the object is present in the arena). To limit the impact of inter-individual variability, data were normalized to a baseline measured for each animal just before the experiment started. Percent change of EEG power corresponds to the power spectrum values for each band of frequencies normalized to the area under the curve of the power spectrum considering frequencies between $1-100 \mathrm{~Hz}$ (except the 48-52 Hz noisy band). Net change of EEG power in the theta band was calculated as follow: (power spectrum in 8-12 Hz band at T1, T4 or T5 - power spectrum in 8-12 Hz band at baseline) - (power spectrum in 4-8 Hz band at T1, T4 or T5 - \% power spectrum in 4-8 Hz band at baseline).

\section{Live slice preparation}

Animals (4-8 weeks old males) were anesthetized using isoflurane and quickly decapitated. Thick coronal slices $(300 \mu \mathrm{m})$ containing the lateral septum were prepared using a vibrating microtome (Campden Instr. Ldt; England) and collected in an ice-cold slicing solution (in mM: $10 \mathrm{NaCl}, 1.2 \mathrm{KCl}, 26 \mathrm{NaHCO} 3,15$ glucose, $1.2 \mathrm{KH}_{2} \mathrm{PO}_{4}, 1 \mathrm{CaCl}_{2}, 2 \mathrm{MgCl}_{2}, 195$ sucrose; osmolality adjusted to $300 \mathrm{mOsmol} .1^{-1} ; \mathrm{pH} 7.4,95 \% \mathrm{O}_{2}$ and $5 \% \mathrm{CO}_{2}$. Slices were allowed to recover for $1 \mathrm{~h}$ at $37^{\circ} \mathrm{C}$ in ACSF medium (in mM: $110 \mathrm{NaCl}, 3.6 \mathrm{KCl}, 26 \mathrm{NaHCO}_{3}, 10$ glucose, $1.2 \mathrm{KH}_{2} \mathrm{PO}_{4}, 2 \mathrm{CaCl}_{2}, 2 \mathrm{MgCl}_{2}, 0.2$ ascorbic acid, 0.2 thio-urea, osmolality adjusted to 300 mOsmol.1 ${ }^{-1} ; \mathrm{pH} 7.4,95 \% \mathrm{O}_{2}$ and $5 \% \mathrm{CO}_{2}$ ) until placed in the recording chamber perfused at $1 \mathrm{~mL} / \mathrm{min}$ with heated ACSF.

\section{Slice electrophysiology}

$\mathrm{An} \mathrm{Ag} / \mathrm{AgCl}$ electrode placed in the recording chamber was used as reference. A similar electrode was inserted in a borosillicated glass (GC150F-10, Harvard apparatus) electrode (4-6 Ohm resistance when immersed the bath) containing intracellular medium (in $\mathrm{mM}: 9 \mathrm{KCl}, 130$ $\mathrm{KMeSO}_{3}, 8 \mathrm{NaCl}, 1 \mathrm{MgCl}_{2}, 0.1 \mathrm{EGTA} / \mathrm{Na}, 10 \mathrm{HEPES} / \mathrm{NaOH}, 2$ pyruvate, 2 malate, 0.5 $\mathrm{NaH}_{2} \mathrm{PO}_{4}, 0.5$ cAMP, 2 ATP-Mg, 0.5 GTP-Tris, 14 phosphocreatine, 0.1 leupeptine) and connected to the pre-amplifier. Recordings were performed using an axopatch $200 \mathrm{~B}$ amplifier (Axon instruments; USA). Neurons visualized using infra-red Normarsky contrast microscopy and recorded from the lateral septum were chosen randomly. Bath application of peptides 
(TGOT $10^{-7} \mathrm{M}$ or vasopressin $10^{-6} \mathrm{M}$ ) was performed in random order for 2 min to determine their impact on electrical/synaptic activity. GABAzine $\left(10^{-6} \mathrm{M}\right)$ or tetrodotoxine (TTX, $3 \times 10^{-7}$ M) were bath-applied for $10 \mathrm{~min}$ at least. Action potentials were recorded in whole-cell or cellattached configurations for at least $5 \mathrm{~min}$ before any drug application, respectively in current or voltage clamp conditions. Interspike intervals (ISI) were calculated over a $3 \mathrm{~min}$ period and classified depending on their duration. Data were normalized to the duration of ISI. AlexaFluor-594-cadaverin (Life technology, $50 \mu \mathrm{M}$ ) was added to the intracellular medium of the patch pipette and whole-cell recordings performed as usual to determine cell morphology $a$ posteriori.

\section{Retrograde tracings}

Alexa-Fluor-555-conjugated latex microspheres (Retrobeads, Lumafluor) diluted 1:1 in PBS were stereotaxically injected in the medial septum (angle $10^{\circ}, \mathrm{AP}+0.05 \mathrm{~cm}, \mathrm{ML}+0.1 \mathrm{~cm}, \mathrm{DV}-$ $0.4 \mathrm{~cm}$ ). After surgeries, mice were group-housed for at least a week before being sacrificed. Brains were processed for slice electrophysiology or immunohistochemistry.

\section{Immunohistochemistry}

Mice were anesthetized with pentobarbital $(50 \mathrm{mg} / \mathrm{kg}$, i.p., Ceva Santé Animale, Libourne, France) and perfused at a rate of $3 \mathrm{ml} / \mathrm{min}$ through the ascending aorta with $30 \mathrm{ml}$ of ice-cold $0.9 \% \mathrm{NaCl}$ and $4 \%$ ice-cold paraformaldehyde. Brains were sectioned with a vibratome and free-floating coronal sections rinsed in PBS were blocked in $3 \%$ normal donkey serum, PBS, $0.1 \%$ triton X-100 for $2 \mathrm{~h}$ at $25{ }^{\circ} \mathrm{C}$. Non-labelled Fab anti mouse IgG (abliance, 1:500) was used to block non-specific sites when mouse antibodies were chosen. Primary antibodies (dilutions for: NG 1:1000, Synaptophysin 1:100, c-Fos 1:1000, p-S6 1:400, Som 1:50, Neurotensin 1:250, GAD67 1:500, NeuN 1:500, Calbindin 1:5000, Calretinin 1:1000, Neurphysin I 1:1000, Neurophysin II 1:500) were incubated overnight. Alexa-Fluor-conjugated secondary antibodies (1:2,000 ThermoFisher Scientific, Waltham, MA, USA) were incubated for $2 \mathrm{~h}$ at $25^{\circ} \mathrm{C}$. Images were acquired with a confocal microscope LSM780 (Carl Zeiss, Iena, Germany) and 10x, 20x dry objectives or 40x oil-immersion objective for co-expression studies and with a epifluorescence microscope (Imager.Z1, axiocam, Carl Zeiss, Iena, Germany). Excitation and acquisition parameters were unchanged during the acquisition of all images.

\section{Image analyses}

More than 67500 cFos cells, 6727 OXT neurons, 5978 AVP neurons, 1665 RB-555 containing cells, and $4491 \mathrm{~d}\left[\right.$ Lys(Alexa-Fluor-647) $\left.{ }^{8}\right] \mathrm{VP}$ labeled cells were counted in all groups to determine proportions of various cells types co-labeled with c-Fos. Images spanning the anterior, central and posterior septum from the same mouse served to count the number of c-Fos positive cells normalized to the surface of the region of interests marked with Image J software $(\mathrm{NIH})$. C-Fos + cells were further averaged between groups of mice for comparison. Reconstruction of the dendritic arborization in Cadaverine-filled cells from Z-stack images ( $1 \mu \mathrm{m}$ steps) and Scholl analysis was performed using Image J. Semi-guided tracing of OXT and AVP fibers into the septum was performed with the NeuronJ plugin. Positions of cannulas and fibers optics were determined under the microscope in at least 3 sections of septum in each postmortem brain. The diffusion areas of the Alexa-594-cadaverine dye were compared in fixed sections of septum between animals to determine a posteriori the diffusion area of drugs with similar molecular weight (e.g. AVP, TGOT, MC, Atosiban). 


\section{Synthesis of $d\left[\right.$ Lys(Alexa-Fluor-647) $\left.{ }^{8}\right] \mathrm{VP}$}

The synthesis of the fluorescent peptide was carried out in the laboratory of Dr M. Manning (University of Toledo, $\mathrm{OH}$ ) as previously described ${ }^{8}$. All solvents and reagents used were analytical grade. Most standard chemicals were purchased from Sigma (St. Louis, MO) or Merck (Darmstadt, Germany). [Deamino-Cys ${ }^{1}$, Lys ${ }^{8}$ ]Vasopressin (Bachem, Bubendorf, Switzerland) $1 \mathrm{mg}$ in $100 \mu \mathrm{L}$ of anhydrous dimethyl formamide and $2.5 \mu \mathrm{L}$ (14 $\mu \mathrm{mol})$ of DIPEA ( $N, N$-diisopropylethylamine) was added to $1 \mathrm{mg}(1.25 \mu \mathrm{mol}, \mathrm{MW} \sim 800)$ of the Alexa-Fluor ${ }^{\circledR}$ 647 carboxylic acid succinimidyl ester (Invitrogen/ThermoFisher Scientific). The reaction mixture was stirred for $5 \mathrm{~h}$ at room temperature in the dark, then mixture was acidified $(6 \mu \mathrm{L}$ TFA), diluted with $10 \mathrm{ml}$ of water and lyophilized. The crude material was diluted with $3 \mathrm{~mL}$ of $0.05 \%$ TFA in water, purified by semi-preparative HPLC on a Hitachi D-7000 HPLC system (detection at $214 \mathrm{~nm}$ ), using a C18 column (218TP510 C18, Vydac hromatograph Grace Vydac, Hesperia, CA), solvent A $0.05 \%$ Tri-Fluoro Acetic acid (TFA) in water, solvent B 0.05\% TFA in acetonitrile, gradient $10-70 \% \mathrm{~B}$ over $30 \mathrm{~min}$, flow rate $5.0 \mathrm{~mL} / \mathrm{min}$. Yield of lyophilized dLVP $1.5 \mathrm{mg}(\sim 87 \%)$, RP-HPLC: $\mathrm{t}_{\mathrm{R}} 15.32 \mathrm{~min}$, purity $100 \%$. The affinity of $\mathrm{d}$ [Lys(Alexa-Fluor$\left.647)^{8}\right]$ VP was tested by competitive binding assays with $[\mathrm{H}]^{3}$ AVP on membrane preparations of HEK293 cells transfected with recombinant mOXTR or mAVPR1B, and liver or kidney to purify endogenous mAVPR1A and AVPR2 as previously described ${ }^{8}$. All assays were run in triplicates in at least $n=3$ independent experiments. The selectivity profile of $d$ [Lys(Alexa-Fluor$647)^{8}$ ]VP is best for mOXTR as its Ki affinity was higher 54, 205 and 2796 times than that of AVPR1B, mAVPR1A and mAVPR2, respectively. A similar strategy was previously used to characterize $\mathrm{V} 1 \mathrm{~b}$ expressing cells in the brain of rats ${ }^{9}$.

\section{Statistics}

Parameters quantified include (i) exploration time with a mouse or an object, (ii) frequency of spikes or synaptic events, (iii) co-expression of d[Lys(Alexa-Fluor-647) $\left.{ }^{8}\right] \mathrm{VP}$ with cell type markers, (iv) position of cannulas, optic fibers and viral-mediated recombination at injection sites, (v) density of c-Fos cells in LS, (vi) change of EEG activity between epochs. Representation of $\mathrm{n}$ for each data set is indicated in figure legends. All data collected in animals were from littermate controls and averaged per experimental groups. Data were considered nonparametric when sample size was moderate. Data were tested for normality and compared to a theorical value with Wilcoxon signed rank test (Prism 8.0 Software, GraphPad). We used a paired t-test for comparing 2 series of values from the same animal or cell. Otherwise we used Mann Whitney t-test. We used Spearman correlation for linear associations between datasets. Data considering one variable and more than 2 conditions were analysed using 1-way ANOVA (Kruskal Wallis). Dunns post-hoc test were used to compare the different groups. We used factorial ANOVA to compare multiple groups (behavior, genotype, optogenetics and pharmacology) followed by post-hoc pairwise comparison with Sidak, Dunnett or Tukey tests for corrections. All data are shown as means \pm standard error of the mean. Significance level is set at $\alpha \leq 0.05$. Randomization: for in vivo pharmacology experiments, animals received the different compounds in a random order. For slice electrophysiology experiments, TGOT or vasopressin were administered in a random order. Inclusion or exclusion criteria: (i) postmortem validation of positions of cannulas, (ii) optic fibers and (iii) viral-mediated recombination. An independent experimenter blind to the conditions performed these analyses. Pre-established criteria for excluding data: (i) misposition of cannulas or fibers optics; (ii) poor recombination of viral-guided tools; (iii) instability of EEG signal. Estimates of sample size were calculated by power analysis based on preliminary data. Sample size was chosen to ensure $80 \%$ power to 
detect the pre-specified effect size. Pre-established criteria for stopping data collection included: (i) mice reaching ethical endpoint limits; (ii) unexpected detachment of cranial implants; (iii) mice interacting $<15 \mathrm{~s}$ during the $1^{\text {st }}$ social encounter or $<10$ times during the $1^{\text {st }}$ encounter with the object; (iv) brains badly perfused and unusable for histology. Complete statistic information can be found in Supplemental Table 1.

\section{Data availability}

Data and reagents can be shared on demand.

\section{Supplemental References}

1. Meziane, H. et al. An Early Postnatal Oxytocin Treatment Prevents Social and Learning Deficits in Adult Mice Deficient for Magel2, a Gene Involved in Prader-Willi Syndrome and Autism. Biol. Psychiatry 78, 85-94 (2015).

2. Tendler, A. \& Wagner, S. Different types of theta rhythmicity are induced by social and fearful stimuli in a network associated with social memory. eLife 4, (2015).

3. Melin, P., Trojnar, J., Johansson, B., Vilhardt, H. \& Akerlund, M. Synthetic antagonists of the myometrial response to vasopressin and oxytocin. J. Endocrinol. 111, 125-131 (1986).

4. Manning, M. et al. Oxytocin and Vasopressin Agonists and Antagonists as Research Tools and Potential Therapeutics. J. Neuroendocrinol. 24, 609-628 (2012).

5. Busnelli, M., Bulgheroni, E., Manning, M., Kleinau, G. \& Chini, B. Selective and potent agonists and antagonists for investigating the role of mouse oxytocin receptors. J. Pharmacol. Exp. Ther. 346, 318-327 (2013).

6. Raam, T., McAvoy, K. M., Besnard, A., Veenema, A. H. \& Sahay, A. Hippocampal oxytocin receptors are necessary for discrimination of social stimuli. Nat. Commun. 8, 2001 (2017).

7. Knobloch, H. S. \& Grinevich, V. Evolution of oxytocin pathways in the brain of vertebrates. Front. Behav. Neurosci. 8, 31 (2014).

8. Corbani, M. et al. Design, synthesis, and pharmacological characterization of fluorescent peptides for imaging human V1b vasopressin or oxytocin receptors. J. Med. Chem. 54, 2864-2877 (2011).

9. Corbani, M. et al. Neuroanatomical distribution and function of the vasopressin V1B receptor in the rat brain deciphered using specific fluorescent ligands. Gen. Comp. Endocrinol. 258, 15-32 (2018).

10. Knight, Z.A. et al. Molecular profiling of activated neurons by phosphorylated ribosome capture. Cell. 151 , 5:1126-37 (2012). 


\section{Supplemental Figures}

A

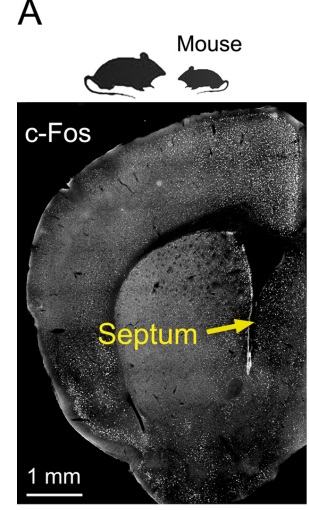

C
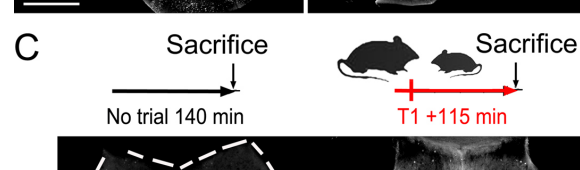

B
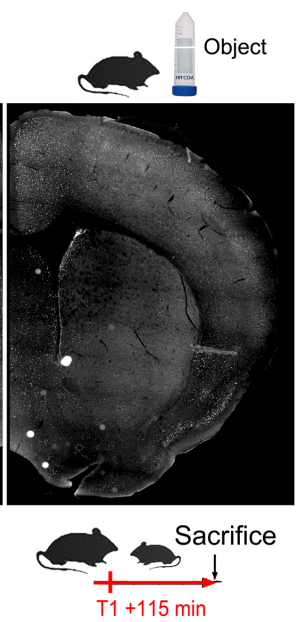

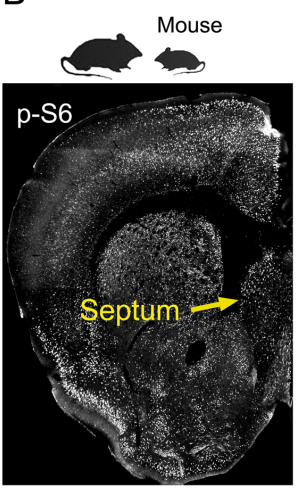

Sacrifice

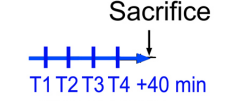

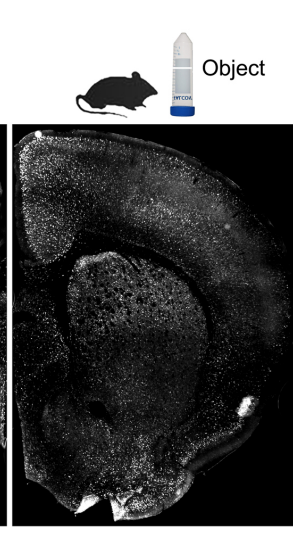

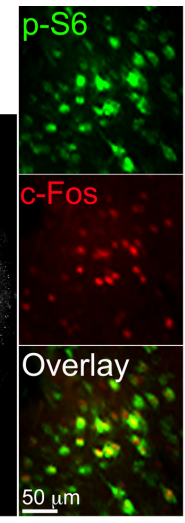

Sacrifice

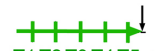

T1 T2 T3 T4 T5 +15 min

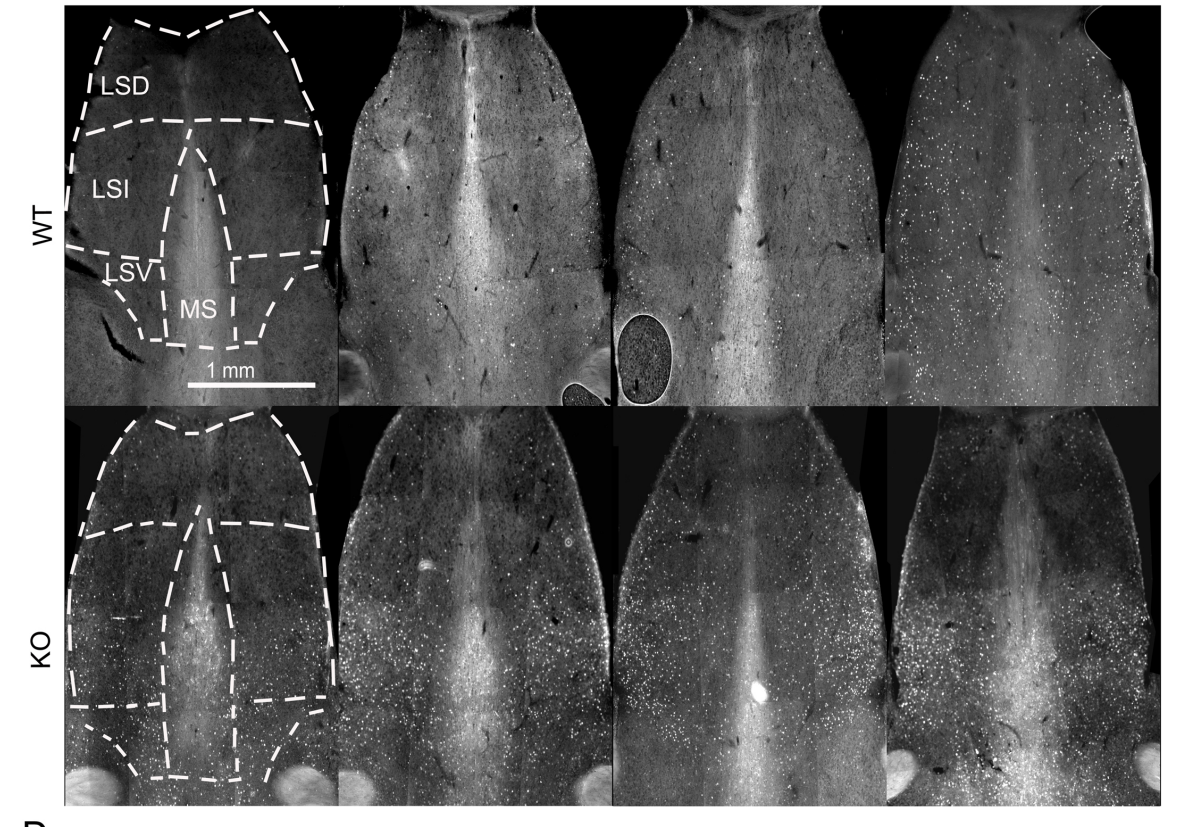

$\mathrm{D}$

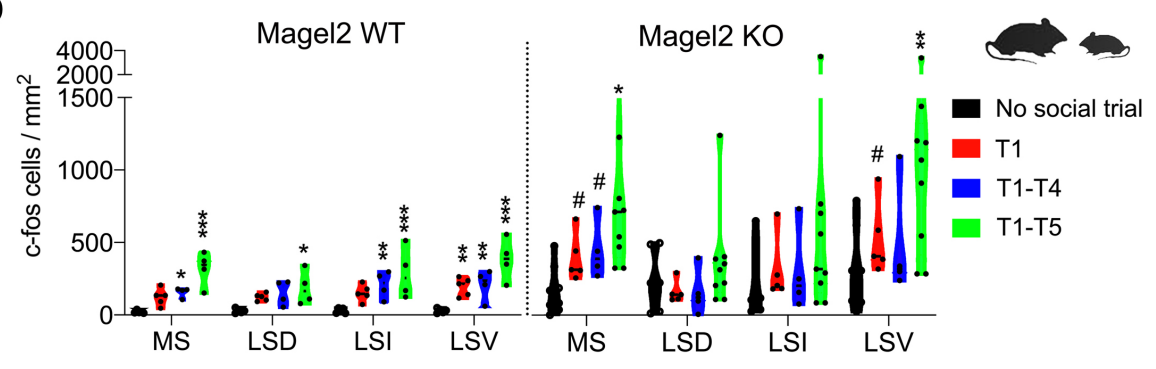

Figure S1. Fos mapping of septum reactivity to social trials of $\mathrm{Magel}^{+m /-p}$ mice with new WT conspecifics.

(a) Representative c-Fos immunoreactivity in a WT mouse interacting with a new WT conspecific for 5 min, sacrificed 15 min later (left) compared to a WT mouse interacting with a new object in the same conditions (right).

(b) Representative p-S6 immunoreactivity in a WT mouse interacting with a new WT conspecific for 5 min, sacrificed 15 min later (left) compared to a WT mouse interacting with a new object in the same conditions (right). 
Colored insets represent a zoom in the dorsal lateral septum co-labeled with c-Fos and p-S6. Same cells responded with both markers in mice sacrificed 15 min after social trial except that signal detection was more robust with an established marker of rapid signaling (p-S6) than a c-Fos, a surrogate of immediate early gene transcription ${ }^{10}$.

(c) Close up look at septal c-Fos immunoreactivity to 1 or more social trials with new conspecifics in Magel2 ${ }^{+m /-p}$ and WT mice as described in Figure 1A. Experimental timelines are as follow: T0 $=140 \mathrm{~min}$ in test chamber before sacrifice; $\mathrm{T} 1=1$ interaction followed by $115 \mathrm{~min}$ in test chamber before sacrifice; T1-T4 $=4$ interactions followed by $40 \mathrm{~min}$ in test chamber before sacrifice; T1-T5 $=5$ interactions followed by $15 \mathrm{~min}$ in test chamber before sacrifice.

(d) Density of c-Fos positive cells $/ \mathrm{mm}^{2}$ in a total of $28072 \mathrm{WT}$ and $38534 \mathrm{KO}$ cells. Means \pm SEM of $\mathrm{n}=6 \mathrm{~T} 0,5$ T1, 4 T4, 4 T5 WT mice and 7 T0, 5 T1, 4 T4, 9 T5 Magel $^{+m / p}$ KO mice. Two-way ANOVA: Effect of social trials in WT: $F(3,56)=31.7, p<0.0001$ post-hoc Dunnett test in MS: T0 vs T5 *** $p<0.0001$ and T0 vs T4 * $p=$ 0.045 ; in LSD: T0 vs T5 $* p=0.016$; in LSI: T0 vs T4 ${ }^{* *} p=0.0063$, T0 vs T5 $* * * p<0.0001$; in LSV: T0 vs T1 ${ }^{* *} p=0.007, \mathrm{~T} 0$ vs $\mathrm{T} 4{ }^{* *} p=0.0058, \mathrm{~T} 0$ vs $\mathrm{T} 5 * * p<0.0001$. Two-way ANOVA: Effect of social trials in KO: $F(3,88)=6.871, p=0.0003$ post-hoc Dunnett test in MS: T0 vs T5 $* p=0.0357$; in LSV: T0 vs T5 $* * p=0.0025$. Multiple comparison between WT and KO by t-test at T1 in MS $t(8)=3.5^{\#} p=0.007$ and LSV $t(8)=2.8^{\#} p=0.02$; at $\mathrm{T} 4$ in $\mathrm{MS} t(6)=2.76^{\#} p=0.032$. 

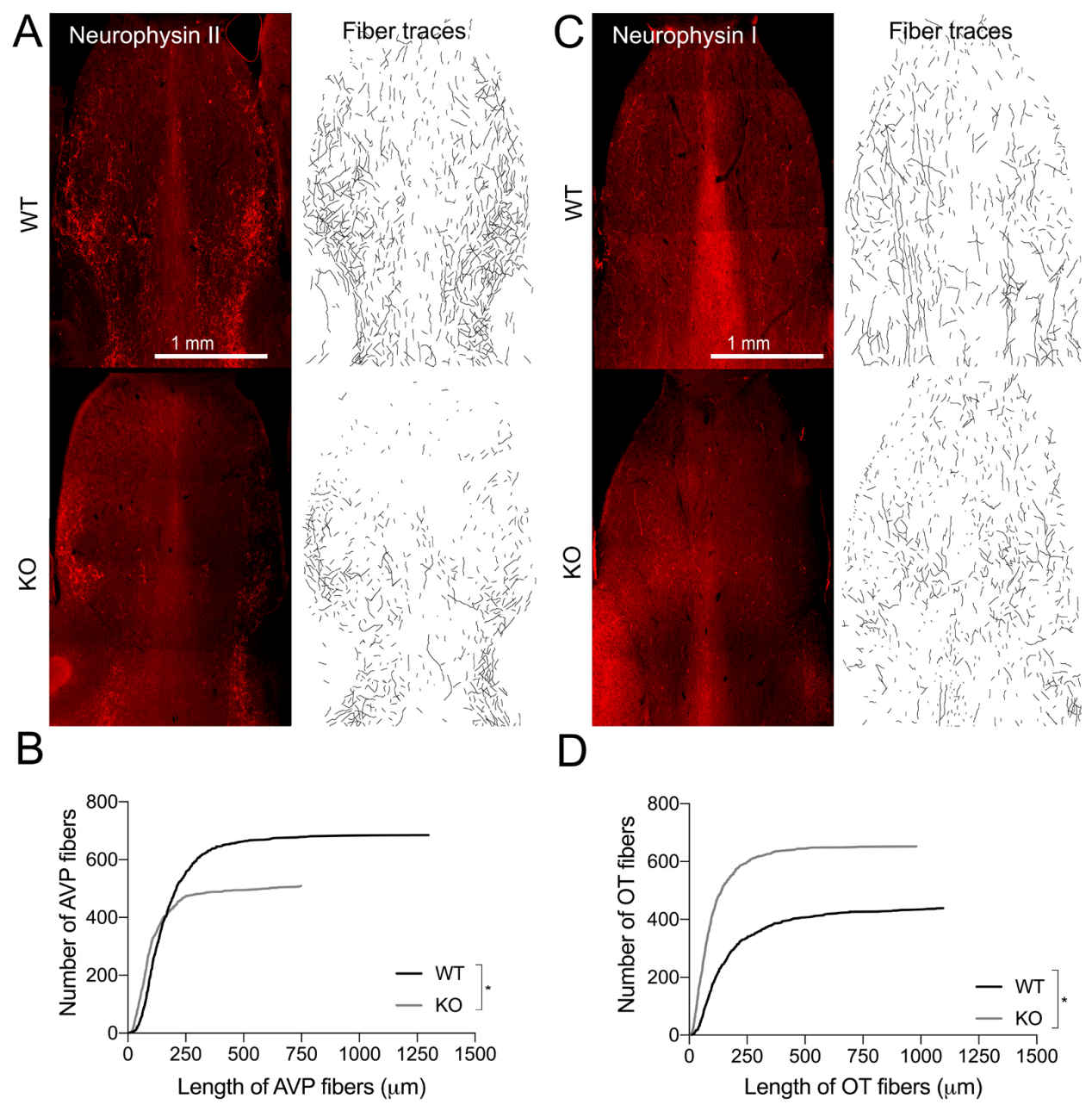

Figure S2. Innervation of AVP and OXT fibers in septum of WT and $\mathrm{Magel2}^{+m /-p} \mathrm{KO}$ mice.

(a) Representative immunoreactivity of neurophysin II (AVP) in septum of WT and Magel2 ${ }^{+m /-p}$ mice. Semiautomated tracing of AVP fibers are shown using NeuronJ.

(b) Number of AVP fibers in $5 \mathrm{WT}$ and $5 \mathrm{KO}$ mice sorted as a function of the length. Kolmogorov-Smirnov test $p$ $<0.0001$.

(c) Representative immunoreactivity of neurophysin I (OXT) in septum of WT and Magel2 ${ }^{+m /-p}$ mice. Semiautomated tracing of OXT fibers are shown using NeuronJ.

(d) Number of OXT fibers in $5 \mathrm{WT}$ and $5 \mathrm{KO}$ mice sorted as a function of the length. Kolmogorov-Smirnov test $p$ $<0.0001$. 


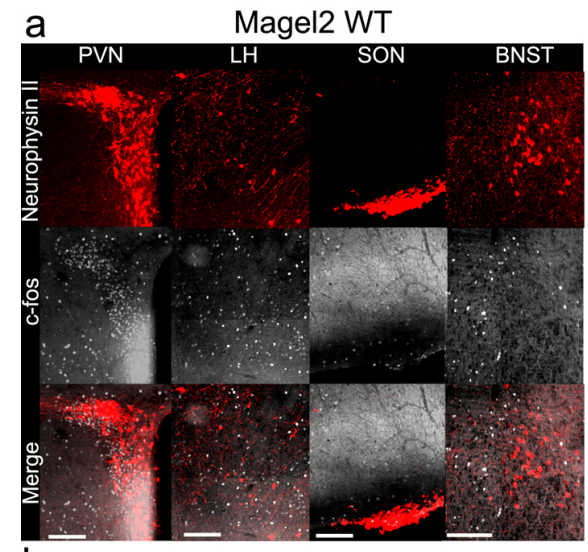

$\mathrm{b}$
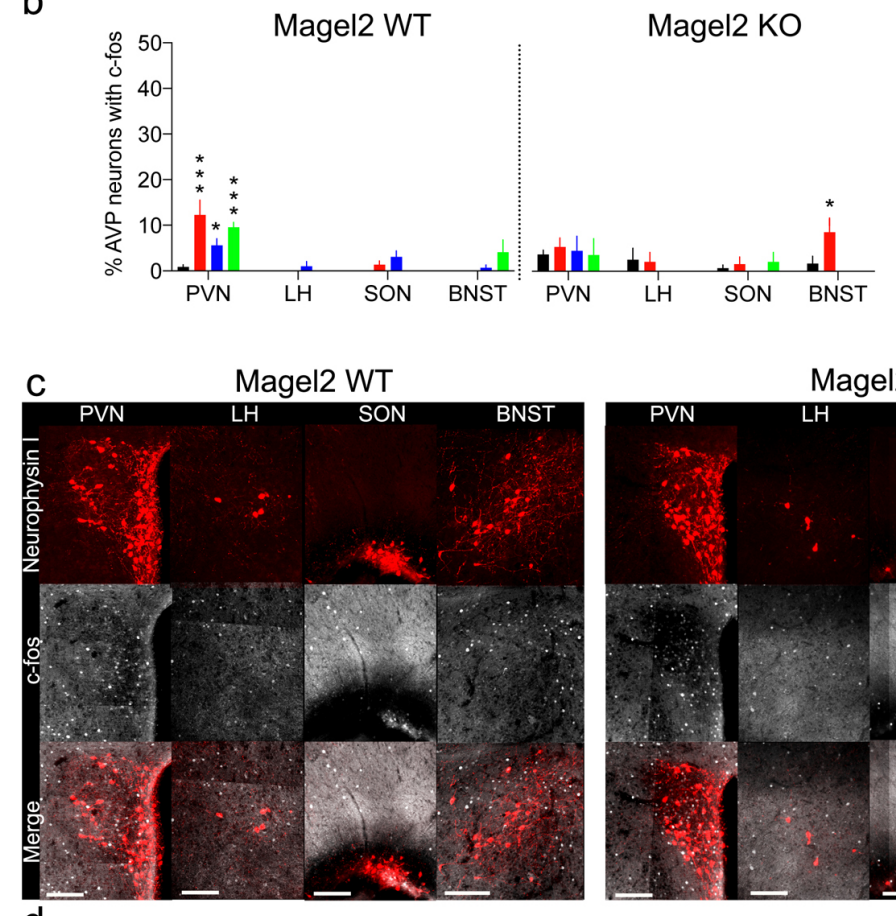

d

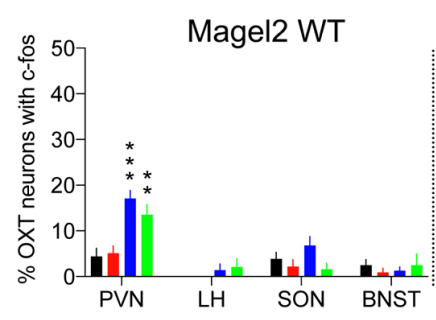

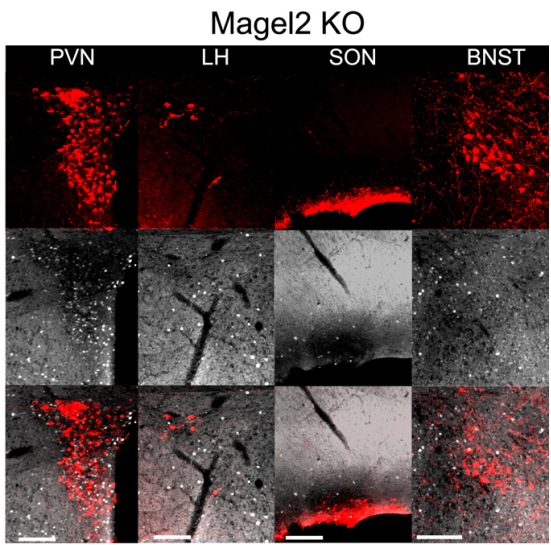

Magel2 KO
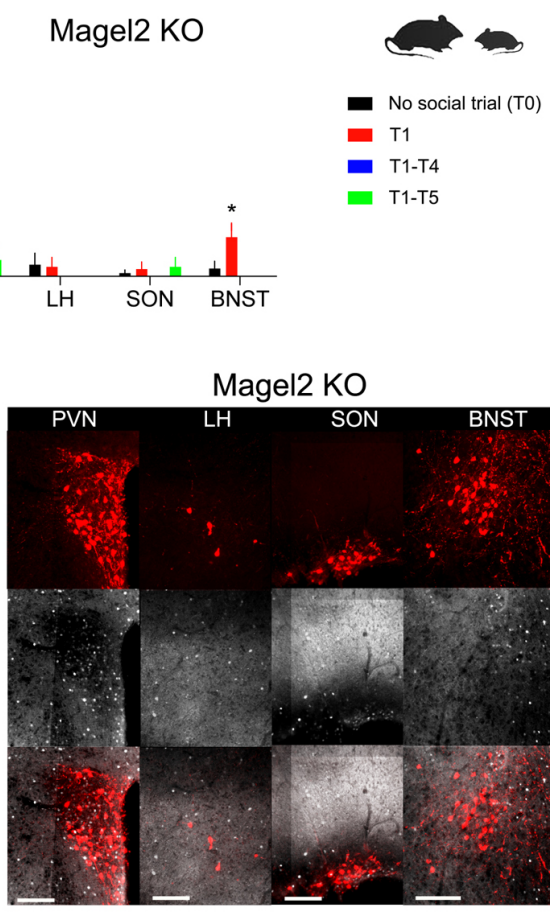

Magel2 KO

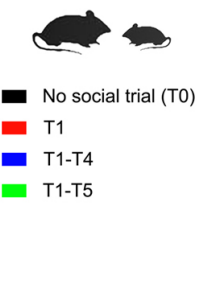

Figure S3. Fos mapping of OXT and AVP neuron reactivity to social trials with new WT conspecifics in Magel2 WT and KO mice.

(a) Induction of c-Fos in AVP neurons (neurophysin II) of paraventricular hypothalamic nucleus (PVN), lateral hypothalamus (LH), supraoptic nucleus (SON) and bed nucleus of stria terminalis (BNST) after 1 social trial (T1) in Magel2 $2^{+m /-p}$ mice and WT controls. Experimental timelines are as follow: $\mathrm{T} 0=140 \mathrm{~min}$ in test chamber before sacrifice; $\mathrm{T} 1=1$ interaction followed by $115 \mathrm{~min}$ in test chamber before sacrifice; T1-T4 $=4$ interactions followed by $40 \mathrm{~min}$ in test chamber before sacrifice; T1-T5 $=5$ interactions followed by $15 \mathrm{~min}$ in test chamber before sacrifice. Scale bars $=50 \mu \mathrm{m}$. 
(b) Percentage of $4133 \mathrm{WT}, 1845 \mathrm{KO}$ AVP neurons expressing c-fos as a function of social trials. Means \pm SEM of $\mathrm{n}=5 \mathrm{~T} 0,5 \mathrm{~T} 1,4 \mathrm{~T} 4,4 \mathrm{~T} 5 \mathrm{WT}$ and Magel2 ${ }^{+m /-p}$ mice. Two-way ANOVA: Effect of social trials on regional AVP neurons in WT: $F(3,56)=24.61, p<0.0001$ post-hoc Dunnett test in PVN: T0 vs T1 $* * * p<0.0001$, T0 vs T5 $* * * p<0.0001$ and T0 vs T4 $* p=0.02$; in KO: $F(3,56)=2.15, p=0.1$ post-hoc Dunnett test in BNST: T0 vs T1 * $p$ $=0.038$.

(c) Induction of c-Fos in OXT neurons (neurophysin I) of PVN, LH, SON and BNST after 4 social trial (T1-T4) in Magel $2^{+m / p}$ mice and WT controls. Scale bars $=50 \mu \mathrm{m}$.

(d) Percentage of $4730 \mathrm{WT}, 1997 \mathrm{KO}$ OXT neurons expressing c-fos as a function of social trials. Means \pm SEM of $\mathrm{n}=5 \mathrm{~T} 0,5 \mathrm{~T} 1,4 \mathrm{~T} 4,4 \mathrm{~T} 5 \mathrm{WT}$ and Magel2 ${ }^{+m /-p}$ mice. Two-way ANOVA: Effect of social trials on regional AVP neurons in WT: $F(3,56)=29.82, p<0.0001$ post-hoc Dunnett test in PVN: T0 vs T4 $* * * p<0.0001$, T0 vs T5 ** $p$ $=0.002$; in $\mathrm{KO}: F(3,56)=3.84, p=0.0143$. 

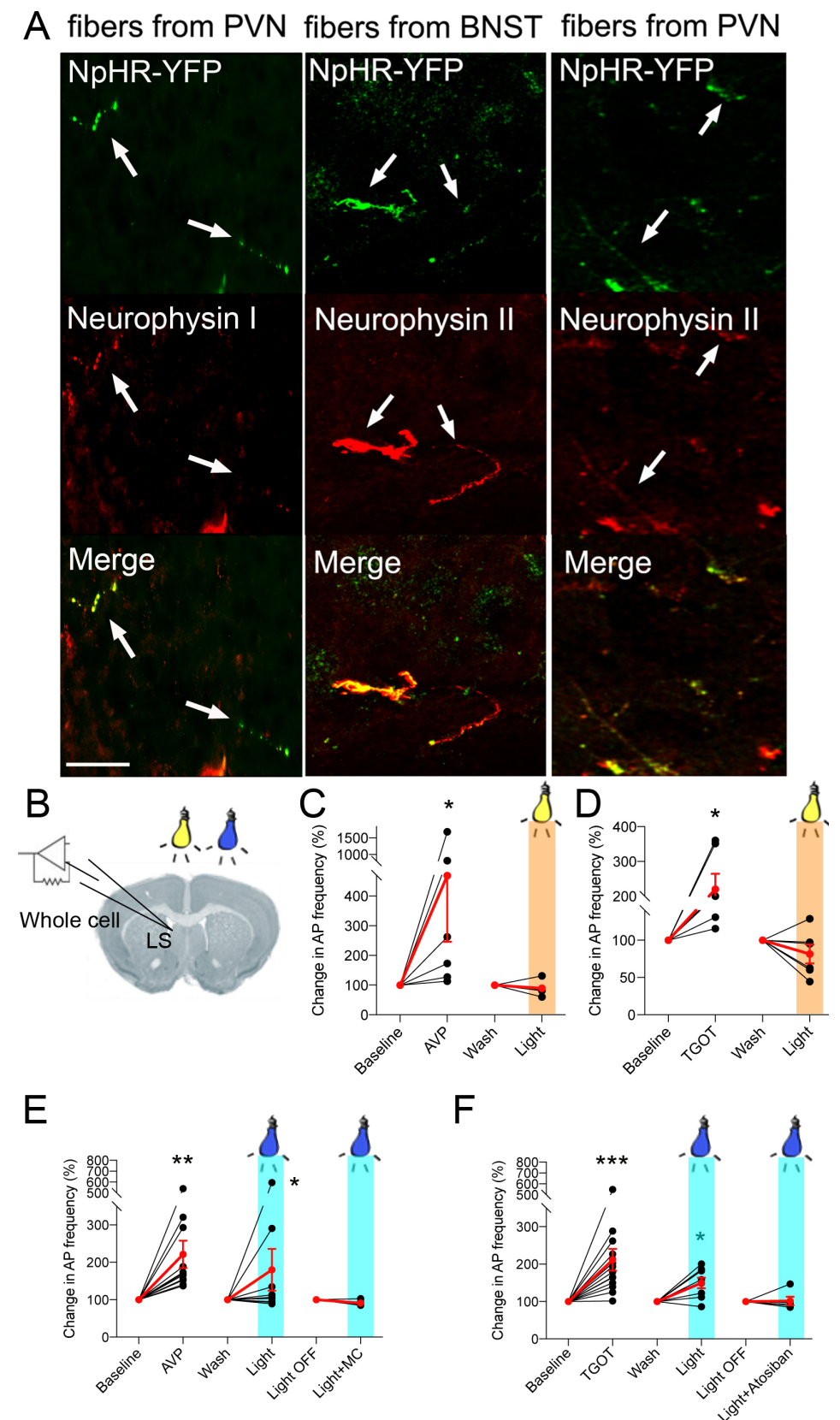

Figure S4. Optogenetic control of AVP and OXT receptor responses in LS.

(a) Viral-mediated expression of NpHR3.0-YFP in PVN to LS fibers of OXT neurons in Oxt-CRE as well as in PVN to LS fibers or BNST to LS fibers of AVP neurons in Avp-CRE mice. Scale $=25 \mu \mathrm{m}$.

(b) Spontaneous activity of LS cells recorded in whole cell configuration in brain slices of recombinant mice.

(c) Effect of yellow light stimulation $(561 \mathrm{~nm}, 5 \mathrm{~ms}$ pulses, $20 \mathrm{~Hz}$ for $2 \mathrm{~min}, \sim 2 \mathrm{~mW}$ ) on the relative frequency of action potentials (AP) of cells pre-identified as AVP-excited $\left(10^{-6} \mathrm{M}, 2 \mathrm{~min}\right) . \mathrm{n}=7$ cells (Means \pm SEM in red): Wilcoxon test $* p=0.015$.

(d) Effect of yellow light stimulation $(561 \mathrm{~nm}, 5 \mathrm{~ms}$ pulses, $20 \mathrm{~Hz}$ for $2 \mathrm{~min}, \sim 2 \mathrm{~mW}$ ) on the relative AP frequency of cells pre-identified as TGOT-excited $\left(10^{-8} \mathrm{M}, 2 \mathrm{~min}\right) . \mathrm{n}=6$ cells (Means \pm SEM in red): Wilcoxon test $* p=$ 0.031 .

(e) Effect of blue light stimulation $(473 \mathrm{~nm}, 20 \mathrm{~Hz}, 5 \mathrm{~ms}$ pulses for $2 \mathrm{~min}, \sim 2 \mathrm{~mW}$ ) on the relative AP frequency of cells pre-identified as AVP-excited $\left(10^{-6} \mathrm{M}, 2 \mathrm{~min}\right) . \mathrm{n}=7$ cells without $\mathrm{MC}$ and 5 cells with $\mathrm{MC}\left(10^{-8} \mathrm{M}\right)(\mathrm{Means} \pm$ SEM in red): Wilcoxon test ${ }^{* *} p=0.001$ and $* p=0.046$. 
(f) Effect of blue light stimulation $(473 \mathrm{~nm}, 30 \mathrm{~Hz}, 10 \mathrm{~ms}$ pulses for $2 \mathrm{~min}, \sim 2 \mathrm{~mW})$ on the relative AP frequency of cells pre-identified as TGOT-excited $\left(10^{-7} \mathrm{M}, 2 \mathrm{~min}\right) . \mathrm{n}=8$ cells without atosiban and 5 cells with atosiban $\left(10^{-8} \mathrm{M}\right)$ (Means \pm SEM in red): Wilcoxon test ${ }^{* * *} p<0.0001$ and ${ }^{*} p=0.02$. 

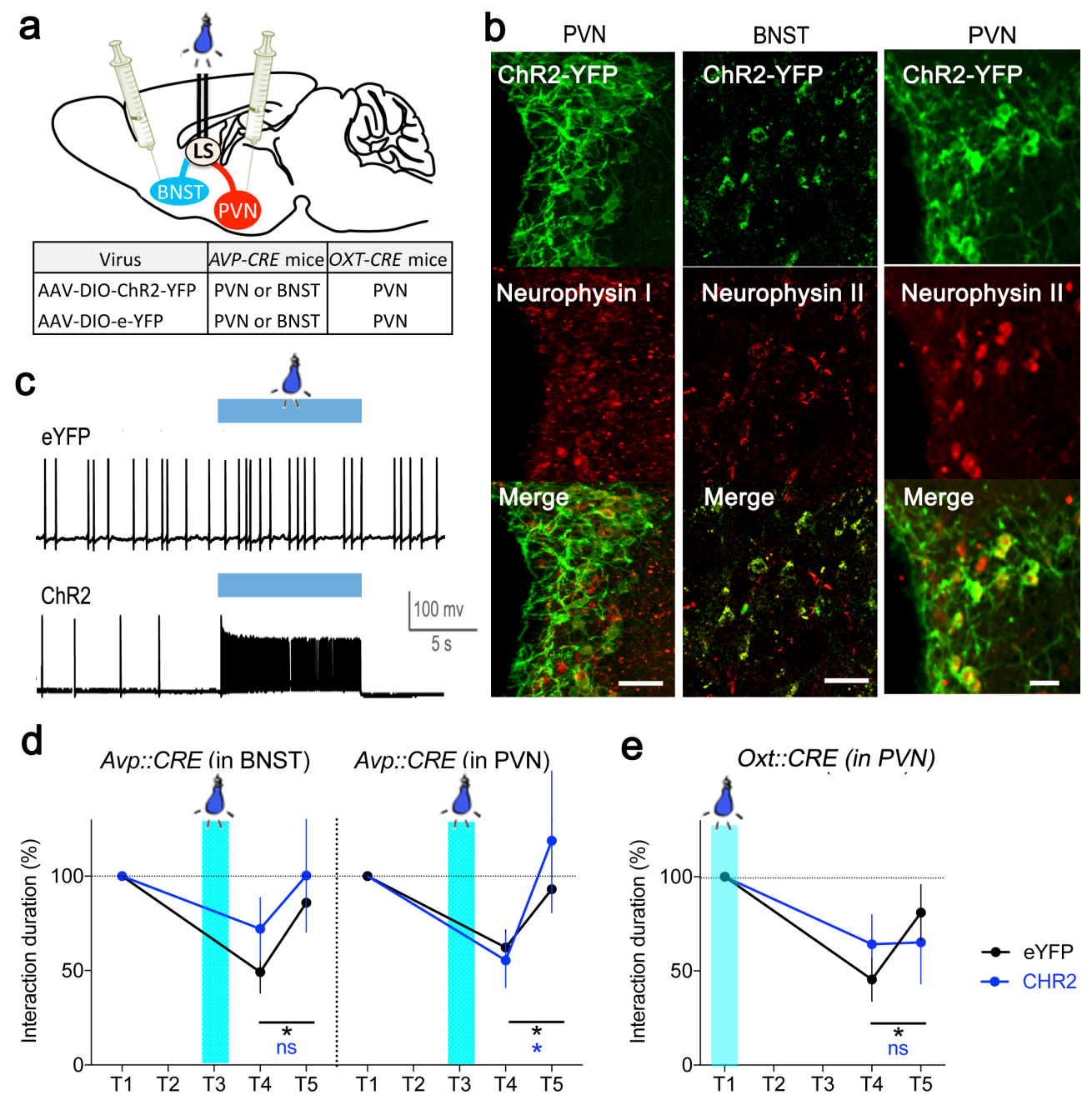

Figure S5. Optogenetic-mediated disorder of AVP and OXT sequential responses in LS impaired social discrimination.

(a) Viral-mediated optogenetic stimulation of septal inputs from OXT neurons (Oxt-CRE mice) in PVN or from AVP neurons (Avp-CRE mice) in PVN or BNST.

(b) Co-expression of Cre-mediated ChR2-YFP with Neurophysin I (OXT) in PVN neurons or with Neurophysin II (AVP) in PVN as well as BNST neurons. Scale $=40 \mu \mathrm{m}$.

(c) Firing of action potentials recorded in whole cell configuration in neurons expressing ChR2-YFP or its eYFP. Stimulation with blue light ( $473 \mathrm{~nm}, 30 \mathrm{~Hz}, 10 \mathrm{~ms}$ pulses for $5 \mathrm{~min}, \sim 2 \mathrm{~mW}$ ) increased firing rate of ChR2 cells.

(d) Percent time interacting with a mouse throughout trials. Data (means \pm SEM) are relative to $\mathrm{T} 1 \mathrm{in}$ each group of $\mathrm{n}=12 \mathrm{eYFP}, 8 \mathrm{ChR} 2$ in BNST and 9 eYFP, $6 \mathrm{ChR} 2$ in PVN of Avp-CRE mice. Two-way ANOVA: Effect of ChR2 stimulation of BNST fibers by blue light $(473 \mathrm{~nm}, 20 \mathrm{~Hz}, 5 \mathrm{~ms}$ pulses for $2 \mathrm{~min}, \sim 2 \mathrm{~mW}$ at T3): $F(2,36)=$ $6.76, p=0.0032$ post-hoc Dunnett test for eYFP controls comparing T4 with $\mathrm{T} 1 * * p=0.0024$, and T4 with T5 $* p=$ 0.028; Effect of ChR2 stimulation of PVN fibers by blue light $(473 \mathrm{~nm}, 20 \mathrm{~Hz}, 5 \mathrm{~ms}$ pulses for $2 \mathrm{~min}, \sim 2 \mathrm{~mW}$ at T3): $F(1,13)=0.2, p=0.65$ post-hoc Dunnett test for eYFP controls comparing T4 with T1 ${ }^{* *} p=0.007$, and T4 with T $5 * p=0.022$; and for ChR2 comparing T4 with T1 ${ }^{*} p=0.046$, and T4 with T5 $p=0.18$.

(e) Percent time interacting with a mouse throughout trials. Data (means \pm SEM) are relative to $\mathrm{T} 1$ in each group of $\mathrm{n}=12 \mathrm{eYFP}, 7 \mathrm{ChR} 2$ in PVN of Oxt-CRE mice stimulated with blue light in LS (473 nm, $30 \mathrm{~Hz}, 10 \mathrm{~ms}$ pulses for 2 $\min , \sim 2 \mathrm{~mW}$ at T3). Two-way ANOVA: Effect of social trials: $F(2,34)=6.77, p=0.0033$, post-hoc Dunnett test for eYFP controls comparing T4 with T1** $p=0.0018$, and T4 with T5 $* p=0.043$. 
A1

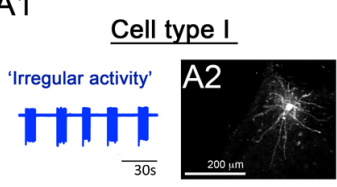

A3

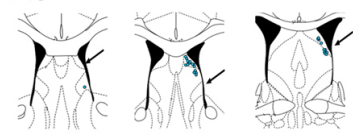

A4

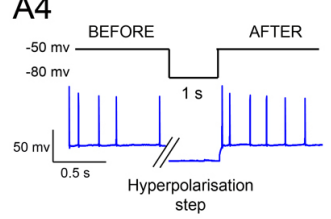

A5

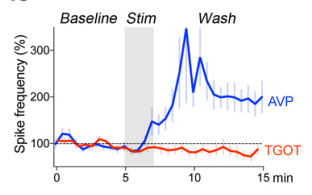

A6

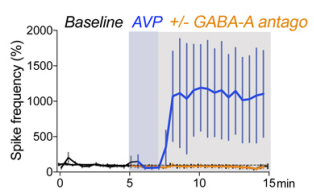

A7
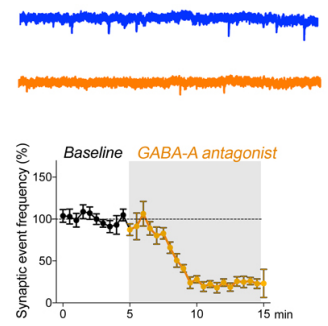

A8

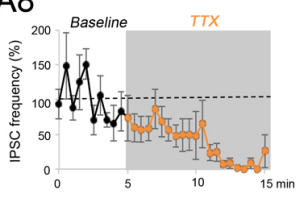

A9

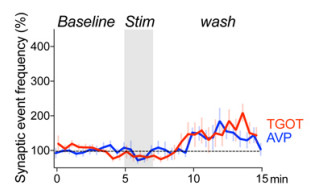

B1

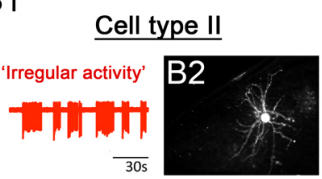

B3

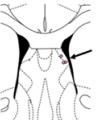

B4

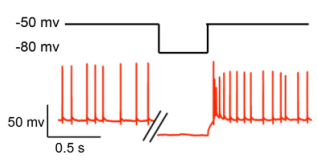

B5

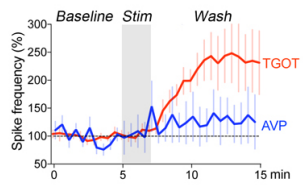

B6

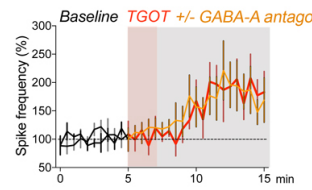

B7
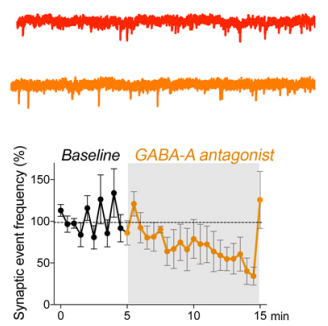

B8

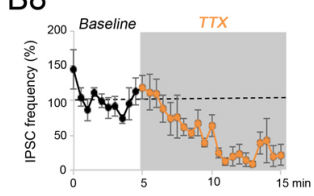

B9

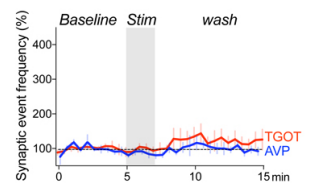

C1

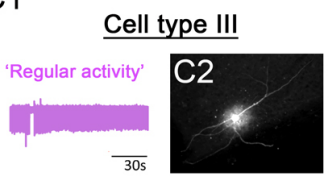

C3

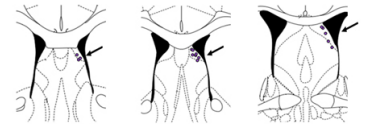

C4

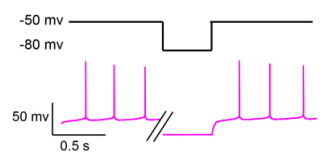

C5

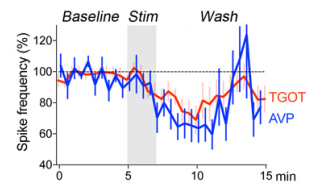

C6

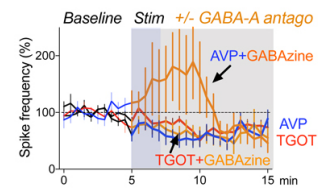

C7

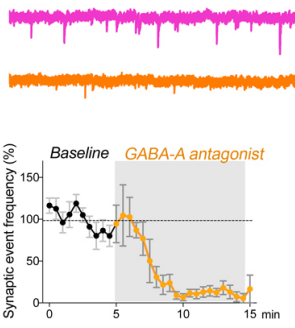

C8

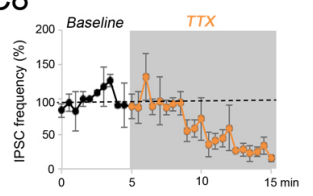

C9

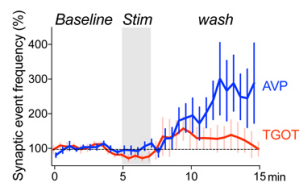

D

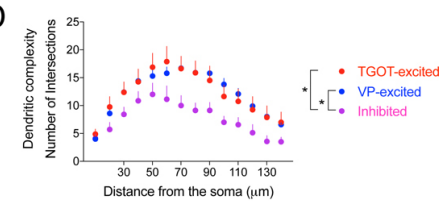

E

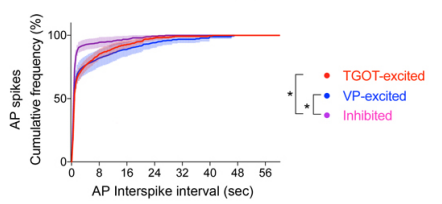

F

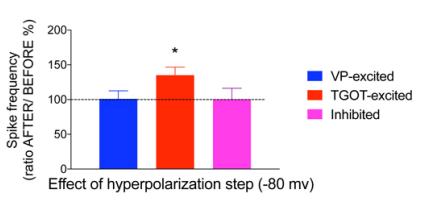

G

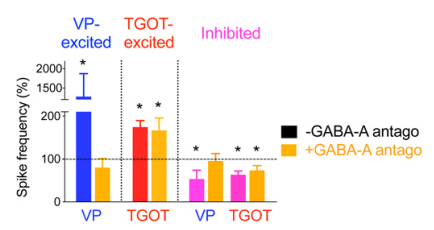

$\mathrm{H}$

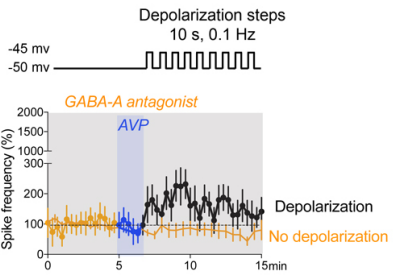

Figure S6. Characterization of 3 major populations of neurons in LS based on their electrophysiological responses to AVPR and OXTR agonists

In brief, type I cells are bursting neurons under strong GABA-A dependent AVP regulation; type II are irregular bursting neurons with a hyperpolarization-activated current whose excitation by TGOT is GABA-A independent; 
and type III neurons exhibit sustained activity decreased by AVP (GABA-A dependent) or by TGOT (GABA-A independent).

(a) Type I: spontaneous spike activity in bursts (A1) of branched cells marked with cadaverine-Alexa594 (A2, see D for group analysis) randomly distributed in LS (A3), excited only by 2 min of $10^{-6}$ M AVP (A5) and completely dependent on GABA-A receptors (A6, see G for group analysis). Synaptic inputs are mostly GABAergic (A7, see I for group analysis) coming from local cells (A8, see $\mathrm{J}$ for group analysis); their frequency is increased by TGOT and AVP (A9, see $\mathrm{K}$ for group analysis).

(b) Type II: spontaneous spike activity irregular in bursts (B1) of branched cells (B2, see D for group analysis) randomly distributed in LS (B3), excited only by $2 \mathrm{~min}$ of $10^{-7} \mathrm{M}$ TGOT (B5) and completely independent on GABA-A receptors (B6, see G for group analysis). Spike activity on these cells is increased by a hyperpolarizationactivated current step (-80 mv, $1 \mathrm{~s}$ ) (B5, see F for group analysis). Synaptic inputs both GABAergic and nonGABAergic (B7, see I for group analysis) most of which come from local cells (B8, see G for group analysis); their frequency is not sensitive to TGOT or AVP (B9, see K for group analysis).

(c) Type III: Regular and sustained spike activity (C1) of tripolar cells (C2, see D for group analysis) randomly distributed in LS (C3), inhibited by AVP and also by TGOT (C5). Spike pattern on these cells is interrupted with silences introduced by AVP and TGOT (C4, see F for group analysis). AVP effect depends on GABA-A receptors contrary to TGOT effect that is insensitive (C6, see G for group analysis). Synaptic inputs are mostly GABAergic (C7, see I for group analysis) coming from local cells (C8, see J for group analysis); their frequency is increased by AVP only (C9, see $\mathrm{K}$ for group analysis).

(d) Morphological characterization of each cell type patched and filled with $50 \mu \mathrm{M}$ cadaverine-Alexa594 for Scholl analysis. Means \pm SEM of $\mathrm{n}=10$ type I cells, 8 type II cells and 7 type III cells. One-way ANOVA: Effect of cell type: $F(2,39)=6.91, p=0.0027$ post-hoc Tukey test: Type I vs type III * $p=0.0072$ and Type II vs type III * $p=$ 0.007 .

(e) Patterns of activity are distinct between cell types. Cumulative frequency of the time during which the cell fires at a frequency defined by each interspike interval epoch. Two-way ANOVA: Effect of cell type: $F(2,12080)=$ $91.19, p<0.0001$ post-hoc Tukey test: ${ }^{*} p<0.0001$.

(f) Bursting activity of type II cells modified by a hyperpolarization-activated current. Hyperpolarization step (-80 $\mathrm{mv}, 1 \mathrm{~s})$ increased spike frequency immediately after repolarization only in type II cells. Means \pm SEM of $\mathrm{n}=20$ type I, 12 type II and 11 type III cells. Paired t-test $t(11)=3.022, * p=0.011$.

(g) Average spike frequency in each cell type. Means \pm SEM of $n=7$ type I, 6 type II and 7 type III cells. Wilcoxon paired t-test: Effect of $10^{-6} \mathrm{M}$ AVP on type I cells $(* p=0.0156)$ lost with GABAzine; No effect of $610^{-6}$ M GABAzine on type II cells stimulated with $10^{-7} \mathrm{M}$ TGOT $\left({ }^{*} p=0.031\right)$; Effect of AVP on type III cells $\left({ }^{*} p=\right.$ $0.046)$ and TGOT on type III cells $(* p=0.015)$ were lost with GABAzine.

(h) Inhibitory effect of $610^{-6} \mathrm{M}$ GABAzine on AVP-mediated excitation of type I cells relieved by small depolarization steps $(+5 \mathrm{mv}, 10 \mathrm{~s}, 0.05 \mathrm{~Hz})$. Therefore, AVP action depends on the existence of small depolarizing events whose impact on neuronal spiking activity is probably increased by AVP (A4). Means \pm SEM of $n=9$ type I cells with depolarization steps and 8 without.

(i) Effect of $610^{-6} \mathrm{M}$ GABAzine on the frequency of synaptic events. Means \pm SEM of $\mathrm{n}=14$ type I, 8 type II and 6 type III cells. Two-way ANOVA: $F(1,50)=44.8, p<0.0001$ post-hoc Sidak test on type I $* * * p<0.0001$, type II ${ }^{*} p=0.05$ and type III $* * p=0.0002$.

(j) Effect of $310^{-7} \mathrm{M}$ TTX on the frequency of synaptic events. Means \pm SEM of $\mathrm{n}=7$ type I, 6 type II and 3 type III cells, paired t-test $* * * p<0.0001,{ }^{* *} p=0.0034, * p=0.013$.

(k) Effect of TGOT and AVP on the frequency of synaptic events in each cell type. $\mathrm{n}=15$ type I, 9 type II and 10 type III cells. Paired t-test for type I $t(9)=3.36,{ }^{* *} p=0.0028$ and $t(9)=2.25,{ }^{*} p=0.04$; type III $t(8)=2.52,{ }^{*} p=$ 0.035 and $t(9)=2.79,{ }^{*} p=0.02$. 


\section{A Intraseptal injection of: $\quad \begin{aligned} & 10 \mu \mathrm{M} \text { d[Lys(Alexa647) } \\ & +5 \mathrm{M} \text { VP to label OXTR } \\ & +5 \mathrm{M} \text { Manning } \mathrm{C} \text { to block AVPR }\end{aligned}$}
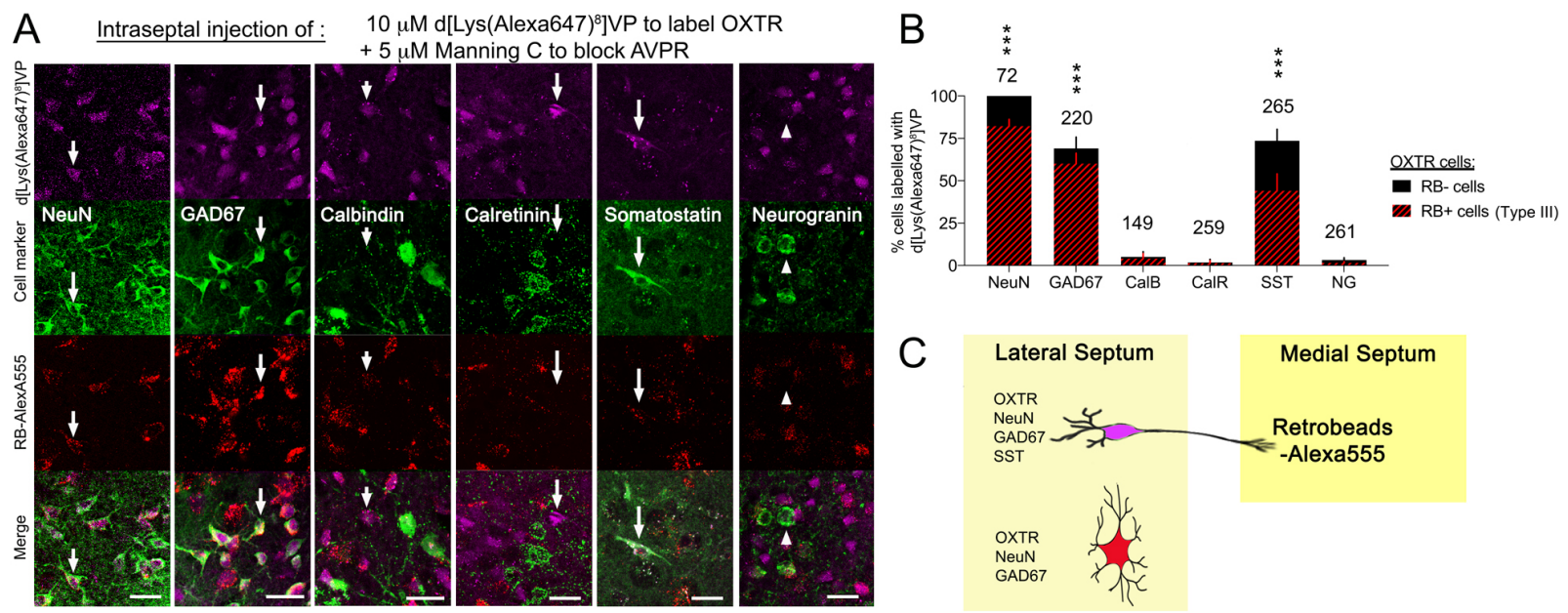

D

Intraseptal injection of

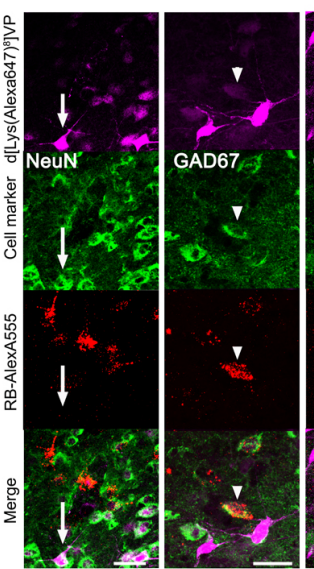

$50 \mu \mathrm{M} d\left[\right.$ Lys(Alexa647) $\left.{ }^{8}\right] \mathrm{VP}$ to label OXTR \& AVPR $+5 \mu \mathrm{M}$ TGOT to block OXTR
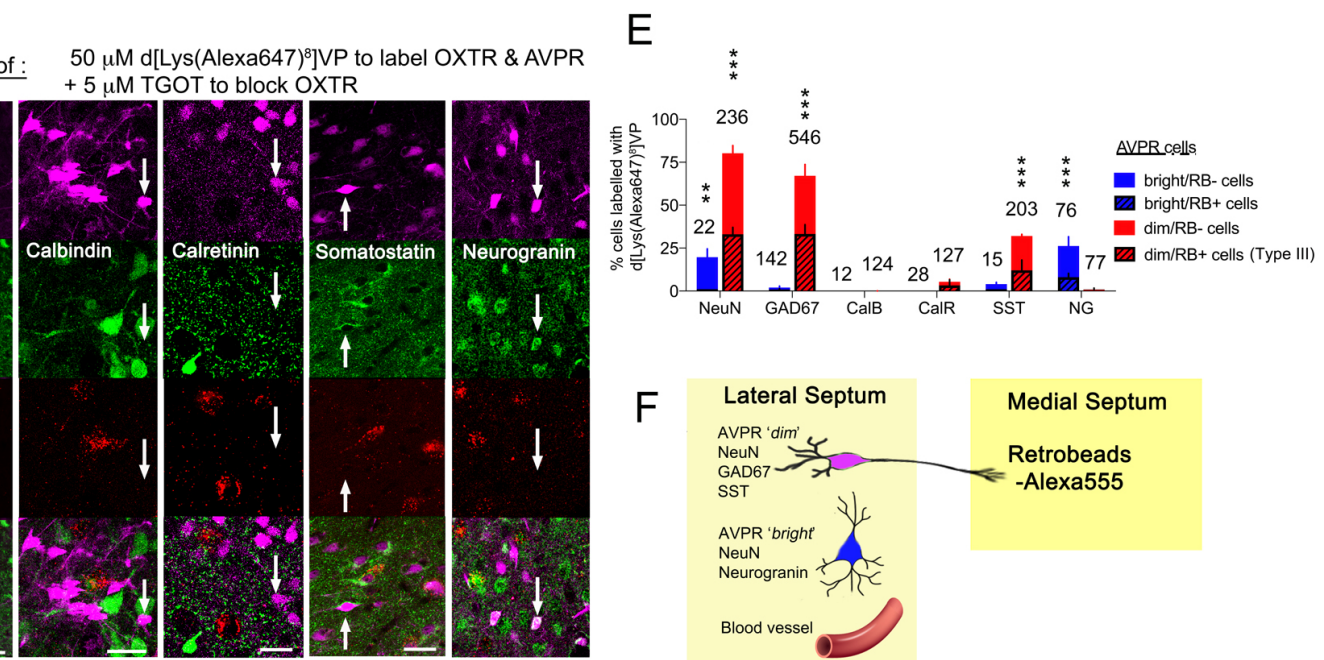

Figure S7. Type III cells are GABAergic neurons projecting to MS, equipped with AVPR and/ or OXTR.

(a) Co-expression of OXTR binding sites [injection of d[Lys(Alexa-Fluor-647) $\left.\left.{ }^{8}\right] \mathrm{VP}+\mathrm{MC}\right]$ with the indicated cellular markers. Arrows point to cells equipped with OXTR. Arrowheads point to cells not equipped with OXTR. Scale bars $=25 \mu \mathrm{m}$.

(b) Percent of cells equipped with OXTR and the indicated marker. Number of cells in specific categories harboring OXTR is indicated out of 1226 OXTR cells of which $334 \mathrm{RB}+$. Wilcoxon unpaired t-test to evaluate the significance of co-expression of OXTR with other markers as well as OXTR/RB + with other markers $* * * p<$ 0.0001 .

(c) LS cells equipped with OXTR are GABAergic/ SST+ and can be sorted in 2 categories: with or without retrobeads.

(d) Co-expression of AVPR binding sites [injection of d[Lys(Alexa-Fluor-647) $\left.{ }^{8} \mathrm{VP}+\mathrm{TGOT}\right]$ with the indicated cellular markers. Arrows point to "bright" cells and arrowheads point to "dim" cells equipped with AVPR. Scale bars $=25 \mu \mathrm{m}$.

(e) Percent of cells equipped with AVPR and the indicated marker out of 1608 AVPR cells of which 523 RB+. Number of cells in specific categories harboring AVPR is indicated. Wilcoxon unpaired t-test to evaluate the significance of co-expression of AVPR with other markers as well as AVPR/RB + with other markers $*^{* *} p<$ $0.0001, * * p<0.001$. 
(f) LS cells equipped with AVPR can be sorted in 4 categories: GABAergic neurons with or without retrobeads and SST, glutamatergic neurons without retrobeads, and blood vessels (see Figure 5b). 

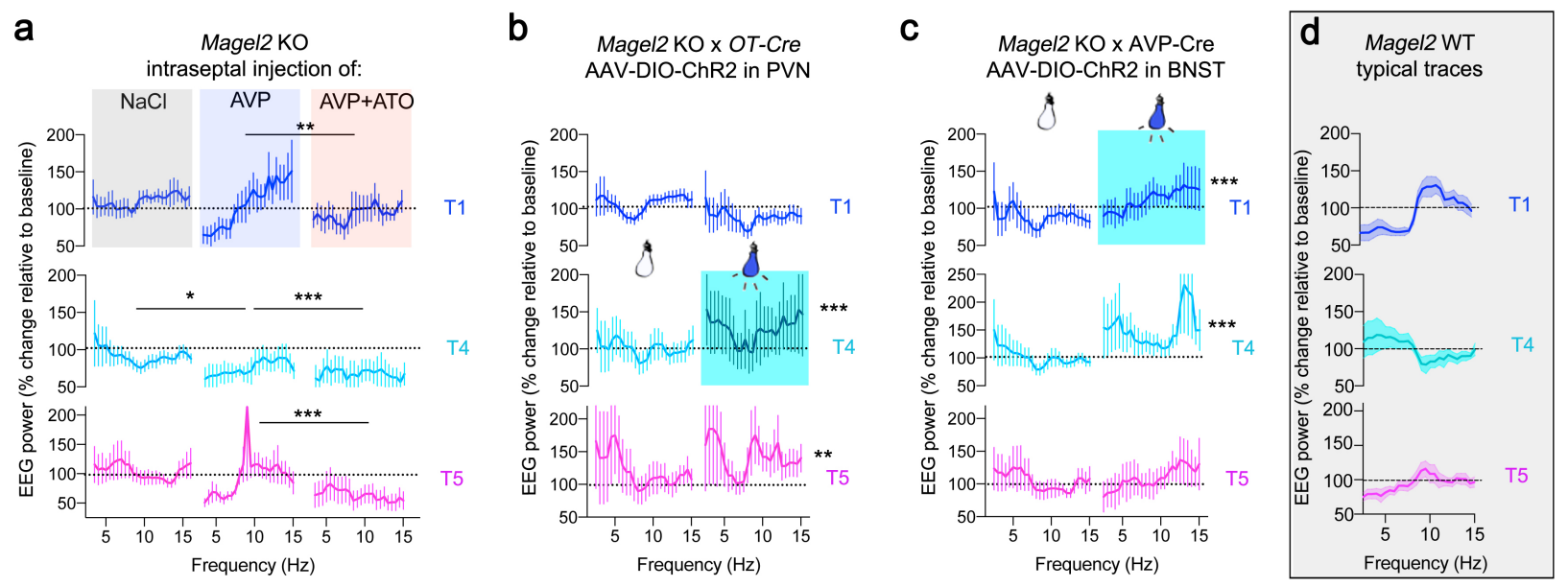

Figure S8. Optogenetic and pharmacological manipulations of $\mathrm{Magel}^{+m /-p} \mathrm{KO}$ EEG activity during social trials.

(a) Percent change of EEG power in 4-12 Hz band upon bilateral injection of $900 \mathrm{~nL}$ of $\mathrm{NaCl}, 3.10^{-6} \mathrm{M}$ AVP with or without $5.10^{-8} \mathrm{M}$ atosiban at $\mathrm{T} 1$ directly in septum of Magel2 $^{+m /-p}$ mice. Means $\pm \mathrm{SEM}$ of $\mathrm{n}=13 \mathrm{NaCl}, 12 \mathrm{AVP}$ and $6 \mathrm{AVP}+$ atosiban mice. Kruskal Wallis test at T1 $t(3)=16.47, p=0.0003$, effect of AVP vs AVP+ATO $* * p=$ 0.0063 ; at $\mathrm{T} 4 t(3)=41.61, p<0.0001$, effect of AVP vs NaCL $* p=0.04$, AVP vs AVP+ATO *** $p<0.0001$; at T5 $t(3)=34.71, p<0.0001$, effect of AVP vs AVP+ATO $* * * p<0.0001$.

(b) Percent change of EEG power in 4-12 Hz band upon bilateral blue light stimulation $(473 \mathrm{~nm}, 30 \mathrm{~Hz}, 10 \mathrm{~ms}$ pulses for $2 \mathrm{~min}, \sim 2 \mathrm{~mW}$ ) in LS of OXT neuron projections between PVN and LS (PVN-LS pathway). Means \pm SEM of $\mathrm{n}=12$ no stim, 12 stim mice. Mann Whitney test at T4 $* * * p<0.0001$, at T5**p 0.0031 .

(c) Percent change of EEG power in 4-12 Hz band upon bilateral blue light stimulation $(473 \mathrm{~nm}, 30 \mathrm{~Hz}, 10 \mathrm{~ms}$ pulses for $2 \mathrm{~min}, \sim 2 \mathrm{~mW}$ ) in LS of AVP neuron projections between BNST and LS (BNST-LS pathway). Means \pm SEM of $\mathrm{n}=9$ no stim, 6 stim mice. Mann Whitney test at T1 and T4 $* * * p<0.0001$.

(d) Typical theta rhythm activity traces socially evoked in control WT mice for comparisons. 
A

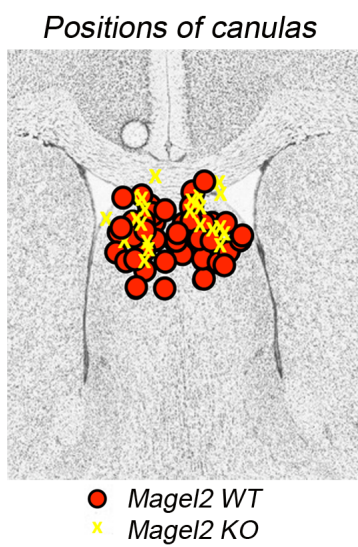

B

Positions of optic fibers

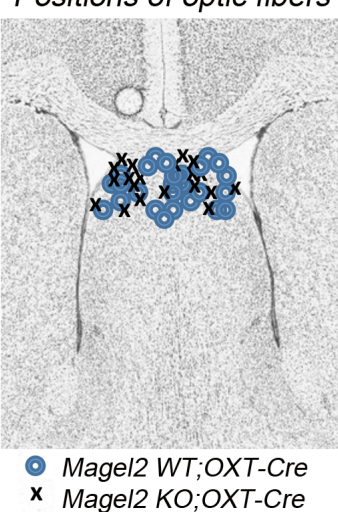

C

Positions of optic fibers

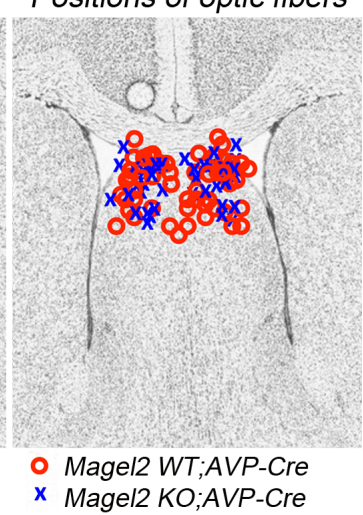

Figure S9. Positions of cannulas and fibers optics in septum of mice included in the study.

(a) Postmortem histological control of the tip of cannulas on each side of LS.

(b) Postmortem histological control of the tip of fiber optics on each side of LS in Oxt-CRE mice.

(c) Postmortem histological control of the tip of fiber optics on each side of $\mathrm{L}$ 